\title{
Mechanics and Quantum Supermechanics of a Monopole Probe Including a Coulomb Potential
}

\author{
Steven G. Avery* and Jeremy Michelson† \\ Department of Physics The Ohio State University \\ 1040 Physics Research Building \\ 191 West Woodruff Avenue \\ Columbus, Ohio 43210-1117 \\ U.S.A.
}

\begin{abstract}
A supersymmetric Lagrangian used to study D-particle probes in a D6-brane background is exactly soluble. We present an analysis of the classical and quantum mechanics of this theory, including classical trajectories in the bosonic theory, and the exact quantum spectrum and wavefunctions, including both bound and unbound states.
\end{abstract}

*Electronic address: avery@mps,ohio-state,edu

${ }^{\dagger}$ Electronic address: jeremy@mps,ohio-state,edu 


\section{Contents}

I. Introduction

II. The Model 4

III. Conserved Quantities $\quad 5$

A. The Classical Symmetry Algebra 6

B. The Quantum Symmetry Algebra 7

IV. Classical Trajectories

V. The Quantum Spectrum $\quad 12$

A. Supersymmetric Ground States $\quad 12$

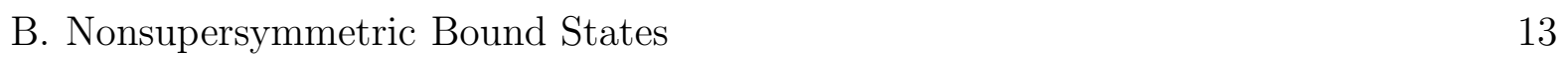

1. Angular Momentum Basis of Nonsupersymmetric Bound States

C. Marginally Bound States $\quad 16$

D. Unbound States $\quad 16$

VI. Wavefunctions $\quad 17$

A. Angular Momentum Eigenfunctions $\quad 17$

B. Ground State Wavefunctions $\quad 19$

C. Bound State Wavefunctions $\quad 20$

1. Bosonic Bound States

2. Fermionic Bound States $\quad 21$

\begin{tabular}{l|l|} 
D. Unbound States & 22 \\
\hline
\end{tabular}

1. Bosonic Unbound States $\quad 22$

2. Fermionic Unbound States $\quad 23$

E. Marginally Bound States $\quad 23$

1. Bosonic States

2. Fermionic States $\quad 24$

Acknowledgments

\begin{tabular}{|l|l|} 
A. Conventions & 24 \\
\hline
\end{tabular}

B. Fermionic Wavefunctions

C. Unitary Representations of $\mathbf{S O}(\mathbf{3}, \mathbf{1})$ and its Contraction 28

D. Normalization of the Unbound Bosonic States

\begin{tabular}{l|l|} 
References & 32 \\
\hline
\end{tabular} 


\section{INTRODUCTION}

The best understanding we have of black holes comes from string theory. Within string theory, those black holes which are constructed by wrapping D-branes on cycles of a compact special holonomy manifold have provided our deepest insights. Nevertheless, there is still much to be understood. For example, while there is a good understanding of fourdimensional black holes obtained by wrapping D2-branes on 2-cycles of a Calabi-Yau 3-fold, an analysis which includes the addition of D6-branes wrapping the entire 3-fold has been elusive.

An additional complication has been the stability properties of D-branes. The appropriate basis of D-brane charges is a function of the Calabi-Yau moduli. Upon crossing lines of marginal stability in moduli space, D-branes may break up into pieces corresponding to the new stable basis. Moreover, the attractor mechanism - or just the fact that the moduli will vary between infinity and the black hole horizon - can mean that lines of marginal stability are crossed in a black hole spacetime, thus leading the microscopic description of the black hole to be more complicated than naively thought.

This paper will not discuss these issues. These issues have been raised and discussed in [1, 2]. One of the consequences of the analysis in [2] is that it becomes interesting to understand the motion of D-particle probes in a D6-brane background. This is equivalently the supersymmetric quantum mechanics of a charged particle in the background of a magnetic monopole. Because D0's and D6's are naively not mutually supersymmetric - though they can form a supersymmetric bound state [3, 4] - the supersymmetric quantum mechanics has a nontrivial potential, which turns out to include a Coulumb potential (see Eq. (II.1) ).

Remarkably, this supersymmetric quantum mechanics is amenable to exact analysis. In particular, in addition to the usual conserved quantities, energy and angular momentum, there is an additional conserved vector quantity. The additional conserved charges are not unlike the Laplace-Runge-Lenz vector found in the hydrogen atom, and so we give it the same name.

It is also interesting that attempting to understand black holes following [2] leads one to study a supersymmetric quantum mechanics. The near horizon limit of a black hole spacetime has an $\mathrm{AdS}_{2}$ factor, whose conformal field theory dual should be a supersymmetric quantum mechanics. [5, 6, 7] Refs. [8, 9] were able to find a supersymmetric quantum mechanics by studying the D-particles induced by D2-branes in the presence of a background Ramond-Ramond field. Interestingly, however, although the supersymmetric quantum mechanics of [6, 7] was, following [10], "Type B" and had fermions which are worldline spinors and target space vectors, the supersymmetric quantum mechanics of [2, 8, 9] is similar to that of [11] in that the fermions are target space spinors.

The purpose of this paper, then, is, with the help of the Laplace-Runge-Lenz vector, to present the exact spectrum and wavefunctions for the theory (II.1). Some of this was already done over 20 years ago, for this theory or for related theories. References include [2, 
12, 13, 14, 15, 16, 17, 18, 19, 20, 21, 22, 23, 24, 25]. However, we have not seen all of the wavefunctions previously, and many of the other results are scattered throughout the literature. So while not all of the results in this paper are new, we have merged our new results with the old results in a self-contained and complete way.

As interesting as it is to have a nontrivial supersymmetric quantum mechanics which is amenable to exact analysis, our ultimate motivation is, of course, an understanding of black holes. We intend for the results presented here to be useful to that end.

It would also be interesting to understand-perhaps similar to the relationship between the Neveu-Schwarz-Ramond superstring and the Green-Schwarz superstring - if the supersymmetric quantum mechanics studied here can be related to those Type B ones studied in e.g. [6, 7].

This paper is organized as follows. The Lagrangian of the model studied in this paper is presented in \$II. The conserved quantities and their classical and quantum algebra are given in $₫ \mathrm{III}$. The classical mechanics is studied in section $₫ \mathrm{IV}$. The quantum spectrum for both bound and unbound states is derived in $\oint \mathrm{V}$. The corresponding wavefunctions are presented in $\oint$ VI. Our conventions are outlined in Appendix A, A derivation of the fermionic superpartners to a bosonic wavefunction is given in Appendix B. An outline of the unitary representations of $\mathrm{SO}(3,1)$ and its contraction, used to study unbound and marginally bound states, is given in Appendix C. Finally, a derivation of the $\delta$-function normalization of the unbound bosonic states is given in Appendix D.

\section{THE MODEL}

In this paper we study the mechanics defined by the Lagrangian

$$
L=\frac{m}{2}\left(\dot{\vec{x}}^{2}+D^{2}+2 i \bar{\lambda} \dot{\lambda}\right)-\left(\frac{\kappa}{2 r}+\theta\right) D-\kappa \vec{A} \cdot \dot{\vec{x}}-\frac{\kappa}{2 r^{3}} \vec{x} \cdot \bar{\lambda} \vec{\sigma} \lambda .
$$

This and related theories have previously been studied in [2, 12, 13, 14, 15, 16, 17, 18, 19, 20, 21, 22, 23, 24, 25]. The Lagrangian (II.1) describes a particle of mass $m$ and integer charge $\kappa$ in the background of a magnetic monopole with unit charge. ${ }^{1}$ There is also a Coulomb potential whose strength is parameterized by the dimensionful (with units of inverse length, in $c=\hbar=1$ units) parameter $\theta$. As the model is a three Euclidean dimensional one, the position of the test particle, $\vec{x}=(x, y, z)$, is a three-vector whose norm is $r=|\vec{x}|$. The fermion $\lambda$ is a two-component spinor whose conjugate is $\bar{\lambda}$; see Appendix $\mathrm{A}$ for conventions. $D$ is an auxiliary field. The vector potential $\vec{A}$ determines the magnetic field $\vec{B}=\vec{\nabla} \times \vec{A}$,

\footnotetext{
${ }^{1}$ Actually, only the product of the electric charge of the test particle and the magnetic charge of the monopole appears in the action. This is $\kappa$.
} 
and is the vector potential for a unit magnetic monople,

$$
\vec{A}=\left\{\begin{array}{ll}
\frac{1}{2}\left(1-\frac{z}{r}\right) \frac{x \hat{y}-y \hat{x}}{x^{2}+y^{2}}, & \frac{z}{r}>-\sin \epsilon, \\
-\frac{1}{2}\left(1+\frac{z}{r}\right) \frac{x \hat{y}-y \hat{x}}{x^{2}+y^{2}}, & \frac{z}{r}<\sin \epsilon,
\end{array} \quad \vec{B}=\vec{\nabla} \times \vec{A}=\frac{1}{2 r^{3}} \vec{x},\right.
$$

in terms of the unit vectors $\hat{x}, \hat{y}, \hat{z}$. The magnetic monopole vector potential is defined in patches which overlap in the region $-\sin \epsilon<\frac{z}{r}<\sin \epsilon, 0<\epsilon<\frac{\pi}{2}$ [26]. The difference between the two vector potentials in the overlap region is pure gauge,

$$
\vec{A}(z>-r \sin \epsilon)-\vec{A}(z<r \sin \epsilon)=\vec{\nabla} \frac{1}{2} \tan ^{-1} \frac{y}{x}, \quad-\sin \epsilon<\frac{z}{r}<\sin \epsilon
$$

Also, the choice of gauge (II.2) gives $\vec{\nabla} \cdot \vec{A}=0$ in both patches.

\section{CONSERVED QUANTITIES}

In terms of the spin angular momentum which is not conserved,

$$
\vec{s}=\frac{m}{2} \bar{\lambda} \vec{\sigma} \lambda
$$

the conserved quantities are

$$
\begin{aligned}
H & =\frac{1}{2 m}(\vec{p}+\kappa \vec{A})^{2}+\frac{m}{2} D^{2}+\frac{\kappa}{m r^{3}} \vec{x} \cdot \vec{s}, \\
\vec{J} & =\vec{x} \times(\vec{p}+\kappa \vec{A})+\frac{\kappa}{2 r} \vec{x}+\vec{s}, \\
\vec{K} & =(\vec{p}+\kappa \vec{A}) \times \vec{J}-i(\vec{p}+\kappa \vec{A})+\frac{\kappa \theta}{2 r} \vec{x}+\frac{\kappa}{r^{3}}(\vec{x} \cdot \vec{s}) \vec{x}-\left(\frac{\kappa}{r}+\theta\right) \vec{s}+(\vec{p}+\kappa \vec{A}) \times \vec{s}, \\
Q & =-m D \lambda-i(\vec{p}+\kappa \vec{A}) \cdot \vec{\sigma} \lambda, \quad \bar{Q}=-m D \bar{\lambda}+i \bar{\lambda} \vec{\sigma} \cdot(\vec{p}+\kappa \vec{A}),
\end{aligned}
$$

These quantities are respectively the Hamiltonian, angular momentum, Laplace-Runge-Lenz vector, and the supercharges. The second term on the right-hand side of (III.4) is a quantum correction, also needed for hermiticity of the operator, and should be omitted classically. Otherwise, these expressions are valid both classically and quantum mechanically. $\vec{p}$ is the canonical momentum $\vec{p}=\frac{\partial L}{\partial \dot{\vec{x}}}$, and the on-shell value of $D$ is

$$
D=\frac{1}{m}\left(\frac{\kappa}{2 r}+\theta\right) .
$$

The first two terms of the angular momenta (III.3) can be considered to be the orbital angular momentum, and the last term is the spin angular momentum. Note, however, that spin angular momentum and orbital angular momentum are not separately conserved.

The Laplace-Runge-Lenz vector (III.4) is the Noether charge associated with the transformation

$$
\begin{aligned}
\delta \vec{x} & =m \vec{x} \times(\vec{\xi} \times \dot{\vec{x}})+m \vec{\xi} \times(\vec{x} \times \dot{\vec{x}})+\frac{\kappa}{2 r} \vec{\xi} \times \vec{x}+2 \vec{\xi} \times \vec{s}, \\
\delta \lambda & =i m \vec{\xi} \cdot(\dot{\vec{x}} \times \vec{\sigma}) \lambda+i \frac{\kappa}{2 r^{3}}(\vec{\xi} \cdot \vec{x})(\vec{x} \cdot \vec{\sigma}) \lambda-\frac{i}{2}\left(\frac{\kappa}{r}+\theta\right) \vec{\xi} \cdot \vec{\sigma} \lambda, \\
\delta D & =-\frac{\kappa}{2 r} \vec{\xi} \cdot \dot{\vec{x}}+\frac{\kappa}{2 r^{3}}(\vec{\xi} \cdot \vec{x})(\vec{x} \cdot \dot{\vec{x}})+\frac{\kappa}{m r^{3}} \vec{\xi} \cdot(\vec{x} \times \vec{s}),
\end{aligned}
$$


parameterized by the vector $\vec{\xi}$. All but the first three terms of the Laplace-Runge-Lenz vector (III.4) are bilinear in the fermions and so vanish in the bosonic theory. The fermion bilinears ensure that $\vec{K}$ is conserved in the supersymmetric theory.

The supercharges (III.5) are the Noether charges associated with the transformation,

$$
\delta \vec{x}=i \bar{\lambda} \vec{\sigma} \xi-i \bar{\xi} \vec{\sigma} \lambda, \quad \delta D=-\dot{\bar{\lambda}} \xi-\bar{\xi} \dot{\lambda}, \quad \delta \lambda=\dot{\vec{x}} \cdot \vec{\sigma} \xi+i D \xi
$$

where the spinor $\xi$ and its conjugate $\bar{\xi}$ parameterize the supersymmetry.

The spinorial supercharges can be combined to form a third conserved vector,

$$
\vec{S}=\frac{1}{4 H} \bar{Q} \vec{\sigma} Q
$$

A convenient normalization has been chosen which, however, only makes the definition (III.9) well-defined away from configurations or quantum states of zero energy. In the nonzero energy sector of the theory, it will be convenient to use modified angular momentum and Laplace-Runge-Lenz vectors

$$
\overrightarrow{\tilde{J}}=\vec{J}-\overrightarrow{\tilde{K}}, \quad \vec{K}+\theta \vec{S}
$$

\section{A. The Classical Symmetry Algebra}

As is standard, the fermions of the theory are first order and therefore they and their momenta are constrained. This is also true of the auxiliary field $D$ and its canonical momentum. It is straightforward to find the nonzero Dirac brackets

$$
\left[x^{i}, p_{j}\right]_{\text {D.B. }}=\delta_{j}^{i}, \quad[\vec{p}, D]_{\text {D.B. }}=\frac{\kappa}{2 m r^{3}} \vec{x}, \quad\left\{\lambda_{\alpha}, \bar{\lambda}^{\beta}\right\}_{\text {D.B. }}=-\frac{i}{m} \delta_{\alpha}^{\beta} .
$$

As a result one finds, for the unmodified quantities, the classical symmetry algebra

$$
\begin{array}{rlrl}
\{Q, Q\}_{\text {D.B. }} & =0=\{\bar{Q}, \bar{Q}\}_{\text {D.B. }}, & \{Q, \bar{Q}\}_{\text {D.B. }} & =-2 i H \mathbb{1}, \\
{[Q, \vec{J}]_{\text {D.B. }}} & =-\frac{i}{2} \vec{\sigma} Q, & {[\bar{Q}, \vec{J}]_{\text {D.B. }}} & =\frac{i}{2} \bar{Q} \vec{\sigma}, \\
{[Q, \vec{K}]_{\text {D.B. }}} & =\frac{i}{2} \theta \vec{\sigma} Q, & {[\bar{Q}, \vec{K}]_{\text {D.B. }}} & =-\frac{i}{2} \theta \bar{Q} \vec{\sigma}, \\
{\left[J^{i}, J^{j}\right]_{\text {D.B. }}} & =\epsilon^{i j k} J^{k}, & {\left[J^{i}, K^{j}\right]_{\text {D.B. }}} & =\epsilon^{i j k} K^{k}, \\
& & & \\
{\left[K^{i}, K^{j}\right]_{\text {D.B. }}=\left(\theta^{2}-2 m H\right) \epsilon^{i j k} J^{k}+\frac{m}{2} \epsilon^{i j k} \bar{Q} \sigma^{k} Q .}
\end{array}
$$

Away from zero energy, we can consider the conserved vector (III.9) and the modified angular momentum and Laplace-Runge-Lenz vectors (III.10). These satisfy an SU(2)×Spin(4), 
$\operatorname{algebra}^{2}$

$$
\begin{aligned}
& {\left[S^{i}, S^{j}\right]_{\text {D.B. }}=\epsilon^{i j k} S^{k}, \quad\left[S^{i}, \tilde{J}^{j}\right]_{\text {D.B. }}=0, \quad\left[S^{i}, \tilde{K}^{j}\right]_{\text {D.B. }}=0,} \\
& {\left[\tilde{J}^{i}, \tilde{J}^{j}\right]_{\text {D.B. }}=\epsilon^{i j k} \tilde{J}^{k}, \quad\left[\tilde{J}^{i}, \tilde{K}^{j}\right]_{\text {D.B. }}=\epsilon^{i j k} \tilde{K}^{k}, \quad\left[\tilde{K}^{i}, \tilde{K}^{j}\right]_{\text {D.B. }}=\left(\theta^{2}-2 m H\right) \epsilon^{i j k} \tilde{J}^{k} \text {. }}
\end{aligned}
$$

The Spin(4) Casimirs are classically given by

$$
\overrightarrow{\tilde{J}} \cdot \overrightarrow{\tilde{K}}=\frac{\kappa^{2} \theta}{4}, \quad\left(2 m H-\theta^{2}\right) \overrightarrow{\tilde{J}}^{2}-\overrightarrow{\tilde{K}}^{2}=-\frac{\kappa^{2}}{2}\left(\theta^{2}-m H\right) .
$$

\section{B. The Quantum Symmetry Algebra}

Quantum mechanically, the Dirac brackets (III.11) become the nonzero commutators

$$
\left[x^{i}, p_{j}\right]=i \delta_{j}^{i}, \quad[\vec{p}, D]=i \frac{\kappa}{2 m r^{3}} \vec{x}, \quad\left\{\lambda_{\alpha}, \bar{\lambda}^{\beta}\right\}=\frac{1}{m} \delta_{\alpha}^{\beta} .
$$

Thus the quantum symmetry algebra is essentially identical to the classical one,

$$
\begin{array}{rlrl}
\{Q, Q\} & =0=\{\bar{Q}, \bar{Q}\}, & \left\{Q_{\alpha}, \bar{Q}^{\beta}\right\} & =2 H \delta_{\alpha}^{\beta}, \\
{[\vec{J}, Q]} & =-\frac{1}{2} \vec{\sigma} Q, & {[\vec{J}, \bar{Q}]} & =\frac{1}{2} \bar{Q} \vec{\sigma}, \\
{[\vec{K}, Q]} & =\frac{\theta}{2} \vec{\sigma} Q, & {[\vec{K}, \bar{Q}]} & =-\frac{\theta}{2} \bar{Q} \vec{\sigma}, \\
{\left[J^{i}, J^{j}\right]} & =i \epsilon^{i j k} J^{k}, & {\left[J^{i}, K^{j}\right]=i \epsilon^{i j k} K^{k},} \\
& {\left[K^{i}, K^{j}\right]=i \epsilon^{i j k}\left[\left(\theta^{2}-2 m H\right) J^{k}+\frac{m}{2} \bar{Q} \sigma^{k} Q\right] .}
\end{array}
$$

Similarly, away from zero energy, ${ }^{3}$

$$
\begin{aligned}
& {\left[S^{i}, S^{j}\right]=i \epsilon^{i j k} S^{k}, \quad\left[\tilde{J}^{i}, S^{j}\right]=0, \quad\left[\tilde{K}^{i}, S^{j}\right]=0,} \\
& {[\vec{S}, Q]=-\frac{1}{2} \vec{\sigma} Q, \quad[\overrightarrow{\tilde{J}}, Q]=0, \quad[\overrightarrow{\tilde{K}}, Q]=0,} \\
& {[\vec{S}, \bar{Q}]=\frac{1}{2} \bar{Q} \vec{\sigma}, \quad[\overrightarrow{\tilde{J}}, \bar{Q}]=0, \quad[\overrightarrow{\tilde{K}}, \bar{Q}]=0,} \\
& {\left[\tilde{J}^{i}, \tilde{J}^{j}\right]=i \epsilon^{i j k} \tilde{J}^{k}, \quad\left[\tilde{J}^{i}, \tilde{K}^{j}\right]=i \epsilon^{i j k} \tilde{K}^{k}, \quad\left[\tilde{K}^{i}, \tilde{K}^{j}\right]=i \epsilon^{i j k}\left(\theta^{2}-2 m H\right) \tilde{J}^{k} .}
\end{aligned}
$$

${ }^{2}$ To be precise, whether the real algebra generated by $\vec{J}$ and $\vec{K}$ is $\operatorname{Spin}(4)$ or $\operatorname{Spin}(3,1)$ depends on whether one is considering the sector of the theory with energies $0<F<\frac{\theta^{2}}{2 m}$ or $F>\frac{\theta^{2}}{2 m}$.

3 These expressions have been given by D'Hoker and Vinet [12, 13, 14, 16, 17], who state that they require a tedius calculation. We agree that they could require a tedius calculation, for we attempted to verify them with Mathematica ${ }^{\circledR}$, but found that $2 \mathrm{~GB}$ of RAM was not enough. Nevertheless, we have at least verified these expressions on constant test spinors. An elegant derivation is given in [21]. 
However, one of the Casimirs is quantum mechanically modified in an important way ${ }^{3}$

$$
\overrightarrow{\tilde{J}} \cdot \overrightarrow{\tilde{K}}=\frac{\kappa^{2} \theta}{4}, \quad \overrightarrow{\tilde{K}}^{2}=\left(2 m H-\theta^{2}\right)\left(\overrightarrow{\widetilde{J}}^{2}+1\right)-\frac{\kappa^{2}}{2}\left(m H-\theta^{2}\right),
$$

Also,

$$
\vec{S}^{2}=\vec{s}^{2}=-\frac{3}{4}\left[(m \bar{\lambda} \lambda)^{2}-2 m \bar{\lambda} \lambda\right]
$$

the second term is a quantum correction.

\section{CLASSICAL TRAJECTORIES}

In this section we consider the classical bosonic theory by setting $\lambda \equiv 0$.

Poincaré [27] has demonstrated that trajectories of charged particles in the presence of a magnetic monopole are always confined to a cone whose tip lies at the monopole. The addition of a radial potential does not effect this. Dotting $\vec{x}$ into Eq. (III.3) yields

$$
\vec{J} \cdot \vec{x}=\frac{\kappa}{2} r .
$$

Spherical symmetry allows us to choose the $z$-axis to be parallel to the angular momentum, so that $\vec{J}=|\vec{J}| \hat{z}$. Then (IV.1) reads

$$
\frac{z}{r}=\frac{\kappa}{2 J}=\text { const }, \quad J \equiv|\vec{J}|,
$$

which is the equation of a cone whose slope is $\frac{\kappa}{\sqrt{4 J^{2}-\kappa^{2}}}$. Thus the dynamics are constrained to the positive (negative) $z$ axis for positive (negative) $\kappa$. Since $z \leq r$, Eq. (IV.2) implies

$$
J \geq \frac{|\kappa|}{2} .
$$

The particular radial potential in this problem admits a conserved Laplace-Runge-Lenz vector which, in this case, restricts the trajectories to also lie in a plane. Without the quantum term in Eq. (III.4), and with the fermions set to zero,

$$
\vec{n} \equiv \vec{J}-\frac{\vec{K}}{\theta}=\left(\vec{x}+\frac{1}{\theta} \vec{J}\right) \times(\vec{p}+\kappa \vec{A}),
$$

is conserved and orthogonal to the velocity vector. Therefore, the trajectories must be confined to the plane whose normal is $\vec{n}$. Thus, the trajectories not only live on the surface of a cone, they are also conic sections; however, the plane of motion does not generally contain the origin.

To be precise, use the Casimirs (III.14) (since the modified vectors coincide with the angular momentum and Laplace-Runge-Lenz vectors when the fermions are set to zero) to see that

$$
|\vec{n}|^{2}=\left|\vec{J}-\frac{\vec{K}}{\theta}\right|^{2}=\frac{2 m H}{\theta^{2}}\left(\vec{J}^{2}-\frac{\kappa^{2}}{4}\right) .
$$


Notice that this is nonzero except for orbits with minimal angular momentum. (Orbits with $J=\frac{\kappa}{2}$ lie entirely on the $z$-axis, by (IV.2).) When $\vec{n}$ is nonzero, the orthogonal distance from the plane of motion to the origin is

$$
z^{\prime} \equiv \vec{x} \cdot \frac{\vec{n}}{|\vec{n}|}=\sqrt{\frac{\vec{J}^{2}-\frac{\kappa^{2}}{4}}{2 m E}}
$$

where $E$ is the energy.

We can complete the definition of the primed coordinates $\left(x^{\prime}, y^{\prime}, z^{\prime}\right)$. First complete the definition of the unprimed coordinates by choosing the $y$ direction (so far only the $z$ direction was chosen) so that the normal vector $\vec{n}$ has no $y$ component. Then choose $x^{\prime}$ to be only a rotation of the $x$ and $z$ directions, i.e.,

$$
\begin{array}{ll}
\hat{x}^{\prime}=\frac{n_{z}}{|\vec{n}|} \hat{x}-\frac{n_{x}}{|\vec{n}|} \hat{z}, & \hat{x}=\frac{n_{z}}{|\vec{n}|} \hat{x}^{\prime}+\frac{n_{x}}{|\vec{n}|} \hat{z}^{\prime}, \\
\hat{y}^{\prime}=\hat{y}, & \hat{y}=\hat{y}^{\prime}, \\
\hat{z}^{\prime}=\frac{\vec{n}}{|\vec{n}|}, & \hat{z}=-\frac{n_{x}}{|\vec{n}|} \hat{x}^{\prime}+\frac{n_{z}}{|\vec{n}|} \hat{z}^{\prime}
\end{array}
$$

Since $\vec{J} \equiv J \hat{z}$,

$$
n_{z}=\frac{\vec{J}}{J} \cdot \vec{n}=J-\frac{\kappa^{2}}{4 J}, \quad n_{x}=\sqrt{\vec{n}^{2}-n_{z}^{2}}=\sqrt{\left(\frac{2 m E}{\theta^{2}}-1+\frac{\kappa^{2}}{4 J^{2}}\right)\left(J^{2}-\frac{\kappa^{2}}{4}\right)} .
$$

It is shown below (see remark 2 on p. 11) that the first factor under the square root is indeed positive, as required for consistency.

Using $r=\sqrt{x^{\prime 2}+y^{\prime 2}+z^{\prime 2}}$ and $z=\left(x^{\prime} \hat{x}^{\prime}+y^{\prime} \hat{y}^{\prime}+z^{\prime} \hat{z}^{\prime}\right) \cdot \hat{z}$ where $z^{\prime}$ is the constant (IV.6), the equation for the cone (IV.2) reads,

$$
\left(1-\epsilon^{2}\right)\left(x^{\prime}-x_{0}^{\prime}\right)^{2}-2 \epsilon r_{0}\left(x^{\prime}-x_{0}^{\prime}\right)+y^{\prime 2}=r_{0}^{2},
$$

where

$$
\epsilon=\sqrt{\frac{2 J^{2}}{\kappa^{2} m E}\left(2 m E-\theta^{2}+\frac{\kappa^{2} \theta^{2}}{4 J^{2}}\right)}, \quad x_{0}^{\prime}=-\frac{\sqrt{J^{2}-\frac{\theta^{2}}{2 m E}\left(J^{2}-\frac{\kappa^{2}}{4}\right)}}{|\theta|-\sqrt{2 m E}}, \quad r_{0}=\frac{4 J^{2}-\kappa^{2}}{2|\kappa| \sqrt{2 m E}} .
$$

This is easily recognized as a conic section in the $\left(x^{\prime}, y^{\prime}\right)$-plane. $\epsilon$ is the eccentricity of the orbit, $r_{0}$ is the semi-latus rectum of the conic section, and the foci are offset along the $x^{\prime}$ axis, with one at $x^{\prime}=x_{0}^{\prime}$ and the other at $x^{\prime}=x_{0}^{\prime}+\frac{2 \epsilon}{1-\epsilon^{2}} r_{0}=-\frac{\sqrt{J^{2}-\frac{\theta^{2}}{2 m E}\left(J^{2}-\frac{\kappa^{2}}{4}\right)}}{|\theta|+\sqrt{2 m E}}$. In the unprimed coordinate system, the foci are located at

$$
\vec{x}=\frac{\sqrt{1-\frac{\theta^{2}}{2 m E}+\frac{\kappa^{2} \theta^{2}}{8 m E}} \sqrt{J^{2}-\frac{\kappa^{2}}{4}}}{\sqrt{2 m E} \pm|\theta|} \hat{x}+\frac{1}{J \sqrt{2 m E}}\left( \pm J^{2}-\frac{\frac{\kappa^{2}}{4}|\theta|}{\sqrt{2 m E} \pm|\theta|}\right) \hat{z} .
$$




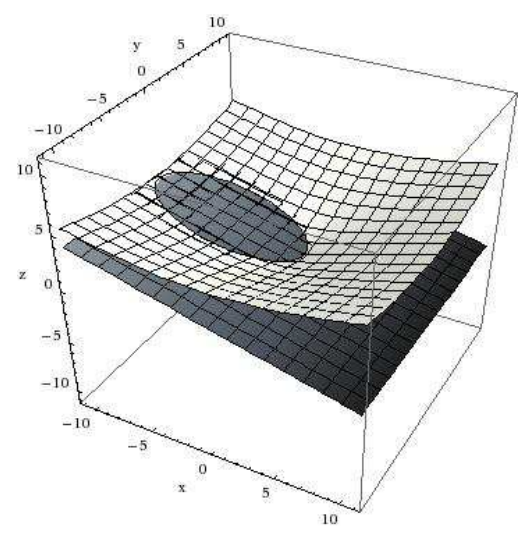

(a)

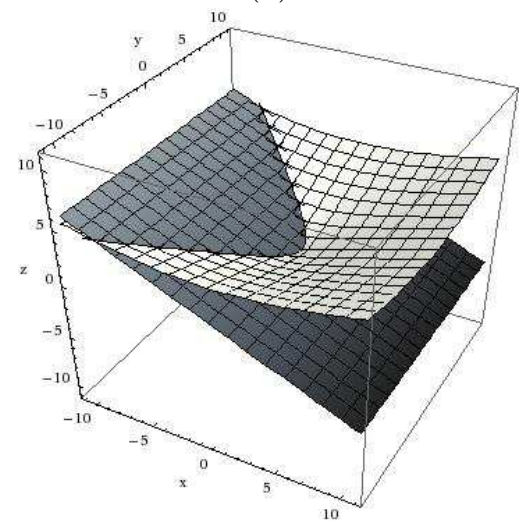

(c)

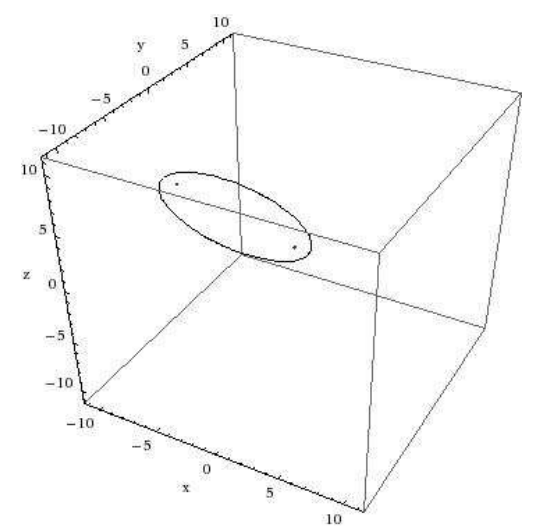

(b)

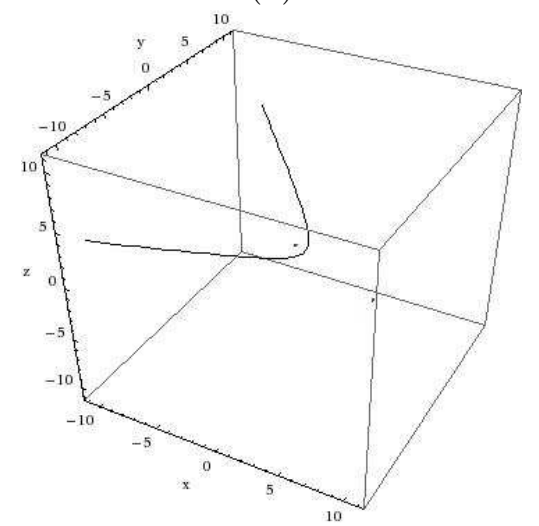

(d)

FIG. 1: Two representative orbits, one bound and one unbound, with $J=\frac{3}{2}, \kappa=1$ and units in which $\theta=-2$. (a) and (b) show a bound orbit with $m E=1.92089$ with and without explicitly exhibiting the plane and the cone on which the orbit lies. In particular, the foci are visible in (b) (c) and (d) are similar with $m E=2.2$.

A bound and an unbound orbit are shown in Fig. 1,

To find the explicit time-dependence of the particle on its orbit, we return to the unprimed coordinates. Upon using cylindrical coordinates $\left(\rho=\sqrt{x^{2}+y^{2}}, \phi=\tan ^{-1} \frac{y}{x}, z\right)$, Eq. (IV.2) yields

$$
\rho(t)=\frac{\sqrt{4 J^{2}-\kappa^{2}}}{\kappa} z(t)=\sqrt{\frac{4 J^{2}}{\kappa^{2}}-1}|z(t)| .
$$

Thus, knowledge of the trajectory reduces to finding the two coordinates $z(t)$ and $\phi(t)$. They are found by using the conserved energy and angular momentum to yield first order differential equations.

Recalling the choice of $z$-axis parallel to $\vec{J}$, the $z$-component of angular momentum is (since $m \dot{\vec{x}}=\vec{p}+\kappa \vec{A}$ )

$$
J=m \rho^{2} \dot{\phi}+\frac{\kappa z}{2 r} \quad \Leftrightarrow \quad z^{2} \dot{\phi}=\frac{\kappa^{2}}{4 m J}
$$


Combining (IV.12) with conservation of energy, $E$, then yields

$$
\dot{z}^{2}=\frac{\kappa^{2}}{4 m^{2} J^{2}}\left[\left(2 m E-\theta^{2}\right)-\frac{\kappa^{2}}{4 z^{2}}-\frac{\kappa^{2} \theta}{2 J z}\right],
$$

which is also valid for $J=\frac{\kappa}{2}$. From (IV.13) we can see a qualitative difference between $E>\frac{\theta^{2}}{2 m}$ and $E<\frac{\theta^{2}}{2 m}$, for the turning points of the motion are located at

$$
z_{\max }=\left[-\frac{\theta}{J} \pm \frac{2}{\kappa} \sqrt{\frac{\kappa^{2} \theta^{2}}{4 J^{2}}+\left(2 m E-\theta^{2}\right)}\right]^{-1} .
$$

Because $z$ and $\kappa$ have the same sign,

1. if $\kappa \theta>0$ then only the choice of plus sign in (IV.14) is valid, and even then only if $2 m E>\theta^{2}$. From (IV.13),$|z|=\infty$ is allowed $\left(\dot{z}^{2} \geq 0\right)$ in this case, so the single turning point gives the minimal value of $z$, and the orbits are unbound.

2. if $\kappa \theta<0$ and $2 m E \leq \theta^{2}$ then both turning points are allowed, and the orbits are bound. Reality of the orbit requires $2 m E \geq \theta^{2}-\frac{\kappa^{2} \theta^{2}}{4 J^{2}} \geq 0$, upon using (IV.3) for the rightmost inequality. The rightmost bound also follows because the Hamiltonian is a sum of squares.

3. if $\kappa \theta<0$ and $2 m E>\theta^{2}$ then only the choice of plus sign in (IV.14) is valid, again corresponding to unbound orbits.

For unbound orbits with $2 m E=\theta^{2}, z_{\min }=\infty$, and so the particle is stuck at infinity.

In any case, the solutions to (IV.12) and (IV.13) are

$$
\phi-\phi_{0}=\cos ^{-1} \frac{|\kappa|(J+\theta z)}{\sqrt{4 J^{2}\left(2 m E-\theta^{2}\right)+\kappa^{2} \theta^{2}} z},
$$

and

$$
\begin{aligned}
t-t_{0}= & \frac{2 m J}{|\kappa|\left(2 m E-\theta^{2}\right)}\left[\sqrt{\left(2 m E-\theta^{2}\right) z^{2}-\frac{\kappa^{2} \theta}{2 J} z-\frac{\kappa^{2}}{4}}\right. \\
& \left.+\frac{\kappa^{2} \theta}{4 J \sqrt{2 m E-\theta^{2}}} \cosh ^{-1} \frac{4 J\left(2 m E-\theta^{2}\right) z-\kappa^{2} \theta}{\sqrt{4 J^{2} \kappa^{2}\left(2 m E-\theta^{2}\right)+\kappa^{4} \theta^{2}}}\right], \quad 2 m E \neq \theta^{2},
\end{aligned}
$$

or

$$
t-t_{0}=\frac{8 J^{2}}{3|\kappa|^{3} \theta^{2}} \sqrt{\frac{\theta}{2 J} z-\frac{1}{4}}\left[\frac{\kappa^{2} \theta}{2 J z-\frac{\kappa^{2}}{4}+3}\right], \quad 2 m E=\theta^{2}
$$

For $2 m E<\theta^{2}$, one can replace $\frac{1}{\sqrt{2 m E-\theta^{2}}} \cosh ^{-1}$ with $\frac{1}{\sqrt{\theta^{2}-2 m E}} \cos ^{-1}$, because Eq. (IV.13) and the inequality in remark 2 above ensure that the arguments of the other square roots are always positive. Moreover, the upper bound from Eq. (IV.14) implies that the argument of the $\cosh ^{-1}$ has magnitude less than one and so should be written as $i \cos ^{-1}$. (For the unbound orbits, the lower bound from Eq. (IV.14) implies that the argument of the $\cosh ^{-1}$ is never less than one.) 


\section{THE QUANTUM SPECTRUM}

The quantum spectrum has been worked out by D'Hoker and Vinet [12, 13, 14, 15, 16, 17]; we repeat the analysis here for notation and completeness. The method is algebraic; the energy spectrum and degeneracy can be extracted from the algebra of the (modified) angular momentum and Laplace-Runge-Lenz vectors.

The Hilbert space is a product of a bosonic Hilbert space and the Hilbert space of the $\lambda$ 's. The latter is defined by choosing the $\lambda$ 's to be annihilation operators and the $\bar{\lambda}$ 's to be creation operators, as per the anticommutation relation (III.15). Using $|00\rangle$ as the "vacuum" state annihilated by both $\lambda_{\alpha}$ 's, the action of $\bar{\lambda}$ creates the other three states

$$
|10\rangle=\sqrt{m} \bar{\lambda}^{1}|00\rangle, \quad|01\rangle=\sqrt{m} \bar{\lambda}^{2}|00\rangle, \quad|11\rangle=m \bar{\lambda}^{1} \bar{\lambda}^{2}|00\rangle=-m \bar{\lambda}^{2} \bar{\lambda}^{1}|00\rangle .
$$

Note the choice of sign for $|11\rangle$ and the normalization which ensures

$$
\left\langle a b \mid a^{\prime} b^{\prime}\right\rangle=\delta_{a a^{\prime}} \delta_{b b^{\prime}}
$$

and

$$
\begin{gathered}
\vec{s}|00\rangle=0=\vec{s}|11\rangle, \\
s^{3}|10\rangle=\frac{1}{2}|10\rangle, \quad s^{+}|10\rangle=0, \quad s^{-}|10\rangle=|01\rangle, \\
s^{3}|01\rangle=-\frac{1}{2}|01\rangle, \quad s^{+}|01\rangle=|10\rangle, \quad s^{-}|01\rangle=0 .
\end{gathered}
$$

The fermion number is $m \bar{\lambda} \lambda$. Although $\vec{s}$ is not conserved by the Hamiltonian, fermion number is a good quantum number.

As was foreshadowed by the introduction of the operators $\vec{S}, \overrightarrow{\tilde{J}}$ and $\overrightarrow{\tilde{K}}$, the analysis will be separated into zero energy and nonzero energy states. Because there is no central charge in the superalgebra (III.16), the supersymmetric states precisely coincide with the states of zero energy and are annihilated by all the supercharges. The states of nonzero energy are never annihilated by a real supercharge (e.g. $Q_{1}+\bar{Q}^{1}$ ), but may be annihilated by a complex supercharge.

\section{A. Supersymmetric Ground States}

Zero energy states - if they exist - are annihilated by $Q$ and by $\bar{Q}$. Eq. (III.16e) shows that on such states,

$$
\vec{N}_{ \pm} \equiv \frac{1}{2}\left(\vec{J} \pm \frac{1}{\theta} \vec{K}\right)
$$

are a pair of canonically normalized commuting $\mathrm{SU}(2)$ generators. One can show that

$$
\vec{J} \cdot \vec{K}=\frac{\theta \kappa^{2}}{4}-m\left(\frac{\kappa}{2 r}+\theta\right) \bar{\lambda} \lambda+m^{2}\left(\frac{\kappa}{2 r}+\frac{3 \theta}{4}\right)(\bar{\lambda} \lambda)^{2}+\frac{m}{2} \vec{x} \cdot[(\vec{p}+\kappa \vec{A}) \times(\bar{\lambda} \vec{\sigma} Q)]+\frac{m}{2} \bar{\lambda} Q
$$


and

$$
\begin{aligned}
& \vec{K}^{2}=\left(2 m H-\theta^{2}\right)\left(\vec{J}^{2}+1\right)-\frac{\kappa^{2}}{2}\left(m H-\theta^{2}\right)+\frac{m^{2}}{2}\left[(\bar{\lambda} \lambda)^{2}-\frac{2}{m} \bar{\lambda} \lambda\right]\left[\theta\left(\frac{2 \kappa}{r}+\theta\right)-5 m H\right]+m \theta\left(\frac{\kappa}{r}+\theta\right) \bar{\lambda} \lambda \\
& +m \theta[\vec{x} \times(\vec{p}+\kappa \vec{A})] \cdot \bar{\lambda} \vec{\sigma} Q+2 m^{2}[\vec{x} \times(\vec{p}+\kappa \vec{A})] \cdot \bar{\lambda} \vec{\sigma} \lambda H-\frac{m^{2} \kappa}{r} \vec{x} \cdot \bar{\lambda} \vec{\sigma} \lambda H+m \theta \bar{\lambda} Q . \quad \text { (V.6) }
\end{aligned}
$$

Thus, on zero energy states

$$
\begin{aligned}
\vec{J} \cdot \vec{K} & =\frac{\theta \kappa^{2}}{4}-\frac{1}{4} m^{2} \theta(\bar{\lambda} \lambda)^{2}+m\left(\frac{\kappa}{2 r}+\theta\right)\left[(\bar{\lambda} \lambda)^{2}-\frac{1}{m} \bar{\lambda} \lambda\right], \\
\vec{K}^{2} & =-\theta^{2}\left(\vec{J}^{2}-\frac{\kappa^{2}}{2}+1\right)+\frac{m \theta^{2}}{2} \bar{\lambda} \lambda+\frac{m^{2} \theta}{2}\left(\frac{2 \kappa}{r}+\theta\right)\left[(\bar{\lambda} \lambda)^{2}-\frac{1}{m} \bar{\lambda} \lambda\right] .
\end{aligned}
$$

Except on states with fermion number 1, these have $r$ dependence, which is incompatible with their conservation. In particular, the Casimirs

$$
\vec{N}_{ \pm}^{2}=(1 \pm 1) \frac{\kappa^{2}}{8}-\frac{1}{4}+\frac{1}{8}(1 \mp 1)+f_{ \pm}(r)\left[(\bar{\lambda} \lambda)^{2}-\frac{1}{m} \bar{\lambda} \lambda\right]
$$

in terms of some functions $f_{ \pm}(r)$ whose precise form we will not need, are not constant except on states with fermion number 1. Therefore, zero energy states must be fermionic.

On the fermionic ground states, then,

$$
\vec{N}_{+}^{2}=\frac{1}{4}(\kappa+1)(\kappa-1), \quad \vec{N}_{-}^{2}=0
$$

corresponding to a singlet under $\vec{N}_{-}$and one multiplet of degeneracy $|\kappa|$ under $\vec{N}_{+} \cdot{ }^{4}$ Since $\vec{J}=\vec{N}_{+}+\vec{N}_{-}$, the ground states thus form a multiplet whose angular momentum is $\frac{|\kappa|-1}{2}$.

\section{B. Nonsupersymmetric Bound States}

To find the spectrum of the remaining bound states, first define the operators

$$
\overrightarrow{\tilde{N}}_{ \pm}=\frac{1}{2}\left(\overrightarrow{\tilde{J}} \pm \frac{1}{\sqrt{\theta^{2}-2 m H}} \overrightarrow{\tilde{K}}\right)
$$

Notice that these operators are Hermitian only on states with $2 m E<\theta^{2}$, where $E$ is the energy. The operators (V.11) are constructed to obey the $\mathrm{SU}(2)^{2}$ algebra

$$
\left[\tilde{N}_{ \pm}^{i}, \tilde{N}_{ \pm}^{j}\right]=\delta_{ \pm \pm^{\prime}} i \epsilon^{i j k} \tilde{N}_{ \pm}^{k}
$$

\footnotetext{
4 To be precise, this argument so far only ensures that the ground states appear in multiples of $|\kappa|$. However, a "highest weight" state will be an eigenstate of $N_{ \pm}^{3}$ - and therefore of $J^{3}$-and be annihilated by $N_{ \pm}^{+}-$ and therefore $J^{+}$and $K^{+}$. This determines the angular part of the state in the multiplet and reduces the determination of the highest weight state to a first order radial differential equation which has at most one normalizable solution. Explicitly solving the wave equation yields one multiplet of ground states when $\kappa \theta<0$ but no zero energy states if $\kappa \theta>0$.
} 
They also commute with the third $\mathrm{SU}(2), \vec{S}$. These three $\mathrm{SU}(2)$ 's are symmetries, and so we can label the states by their quantum numbers, though we will see that they are not completely independent.

Upon using Eq. (ㅍI.14), the Casimirs of $\overrightarrow{\tilde{N}}_{ \pm}$are

$$
\overrightarrow{\tilde{N}}_{ \pm}^{2}=-\frac{1}{4}+\frac{\kappa^{2}}{16}\left(-\frac{\theta}{\sqrt{\theta^{2}-2 m H}} \mp 1\right)^{2}
$$

But of course, on a given $\mathrm{SU}(2)^{2}$ state, $\overrightarrow{\tilde{N}}_{ \pm}^{2}=n_{ \pm}\left(n_{ \pm}+1\right)=\left(n_{ \pm}+\frac{1}{2}\right)^{2}-\frac{1}{4}$, with $n_{ \pm}$halfinteger. Thus,

$$
n_{ \pm}+\frac{1}{2}=\frac{|\kappa|}{4}\left(-\frac{\theta}{\sqrt{\theta^{2}-2 m E}} \mp 1\right) .
$$

Therefore,

$$
n_{-}-n_{+}=\frac{|\kappa|}{2}
$$

In particular, $n_{-}$is larger than $n_{+}$. Also,

$$
n_{+}+n_{-}+1=2 n_{-}-\frac{|\kappa|}{2}+1=-\frac{|\kappa| \theta}{2 \sqrt{\theta^{2}-2 m E}}
$$

from which we read off the energy

$$
2 m E=\frac{4 n \theta^{2}(n-|\kappa|)}{(2 n-|\kappa|)^{2}}, \quad n \equiv 2 n_{-}+1 .
$$

Eq. (V.17) defines the positive, integer quantum number $n$. Since $n_{-}=n_{+}+\frac{|\kappa|}{2}$ and $n_{+}$is positive semi-definite,

$$
n=|\kappa|+1,|\kappa|+2, \ldots .
$$

So far only $\vec{N}_{ \pm}$, has been used and not $\vec{S}$. Because $\vec{S}$ commutes with $\vec{N}_{ \pm}$and the Casimir (III.19) only depends on the fermion number, fermion number is a good simultaneous quantum number. In particular, consider the nonzero bound states with fermion number zero. These are denoted by

$$
\begin{array}{ll}
\left|n ; 00 ; n_{3}^{-}, n_{3}^{+}\right\rangle, \quad & n_{3}^{-}=-\frac{n-1}{2},-\frac{n-1}{2}+1, \ldots, \frac{n-1}{2}-1, \frac{n-1}{2} \\
& n_{3}^{+}=-\frac{n-|\kappa|-1}{2},-\frac{n-|\kappa|-1}{2}+1, \ldots, \frac{n-|\kappa|-1}{2}-1, \frac{n-|\kappa|-1}{2} .
\end{array}
$$

These states are annihilated by both $Q$ s. The $\bar{Q}$ s generate three more sets of states,

$$
\begin{gathered}
\left|n ; 10 ; n_{3}^{-}, n_{3}^{+}\right\rangle \equiv \frac{1}{\sqrt{2 m E}} \bar{Q}^{1}\left|n ; 00 ; n_{3}^{-}, n_{3}^{+}\right\rangle, \quad\left|n ; 01 ; n_{3}^{-}, n_{3}^{+}\right\rangle \equiv \frac{1}{\sqrt{2 m E}} \bar{Q}^{2}\left|n ; 00 ; n_{3}^{-}, n_{3}^{+}\right\rangle, \\
\left|n ; 11 ; n_{3}^{-}, n_{3}^{+}\right\rangle \equiv \frac{1}{2 m E} \bar{Q}^{1} \bar{Q}^{2}\left|n ; 00 ; n_{3}^{-}, n_{3}^{+}\right\rangle .
\end{gathered}
$$


The states $\left|n ; \sigma_{1} \sigma_{2} ; n_{3}^{-}, n_{3}^{+}\right\rangle$consist of two singlets and a doublet under $\vec{S}^{5}$; a multiplet of degeneracy $n$ under $\vec{N}_{-}$; and a multiplet of degeneracy $n-|\kappa|$ under $\vec{N}_{+}$. Thus, the total degeneracy of the energy level (V.17) is

$$
\text { degeneracy }=4 n(n-|\kappa|) \text {. }
$$

As in footnote 4 (p. 13), it has really only been shown that the bound states at level $n$ come in multiples of $4 n(n-|\kappa|)$, and in fact there are no bound states if $\kappa \theta>0$, but there are indeed precisely $4 n(n-|\kappa|)$ states with quantum number $n$ if $\kappa \theta<0$.

\section{Angular Momentum Basis of Nonsupersymmetric Bound States}

The basis (V.19) is not very physical because angular momentum is not well-defined in that basis. This is easily fixed. Because

$$
\vec{J}=\overrightarrow{\tilde{J}}+\vec{S}=\overrightarrow{\tilde{N}}_{+}+\overrightarrow{\tilde{N}}_{-}+\vec{S}
$$

the basis adapted to the angular momentum is a simple application of the rules for summing angular momentum. In particular, since $\vec{S}$ does not commute with $\vec{J}$, it is not possible to specify both, but it is still possible to simultaneously specify the fermion number with the angular momentum. The resulting states are therefore

$$
\begin{aligned}
& \tilde{\jmath}=\frac{|\kappa|}{2}, \frac{|\kappa|}{2}+1, \ldots, n-\frac{|\kappa|}{2}-2, n-\frac{|\kappa|}{2}-1, \\
&\left|n ; \sigma_{1}+\sigma_{2} ; \tilde{\jmath}, j, j_{3}\right\rangle, j= \begin{cases}\tilde{\jmath}, & \sigma_{1}+\sigma_{2}=0,2, \\
\tilde{\jmath} \pm \frac{1}{2}, & \sigma_{1}+\sigma_{2}=1,\end{cases} \\
& j_{3}=-j,-j+1, \ldots, j-1, j,
\end{aligned}
$$

where $\sigma_{1}+\sigma_{2}$ is the fermion occupation number, and $\overrightarrow{\tilde{J}}^{2}=\tilde{\jmath}(\tilde{\jmath}+1)$.

It is straightforward to use Clebsch-Gordan coefficients to find the fermionic states from the bosonic ones. Specifically,

$$
\begin{aligned}
\left|n ; 1 ; j-\frac{1}{2}, j, j_{3}\right\rangle= & \frac{1}{\sqrt{4 j E}}\left[\sqrt{j+j_{3}} \bar{Q}^{1}\left|n ; 0 ; j-\frac{1}{2}, j-\frac{1}{2}, j_{3}-\frac{1}{2}\right\rangle\right. \\
& \left.+\sqrt{j-j_{3}} \bar{Q}^{2}\left|n ; 0 ; j-\frac{1}{2}, j-\frac{1}{2}, j_{3}+\frac{1}{2}\right\rangle\right], \quad j=\frac{|\kappa|+1}{2}, \frac{|\kappa|+3}{2}, \ldots, n-\frac{|\kappa|+1}{2} . \\
\left|n ; 1 ; j+\frac{1}{2}, j, j_{3}\right\rangle= & \frac{1}{\sqrt{4(j+1) E}}\left[-\sqrt{j-j_{3}+1} \bar{Q}^{1}\left|n ; 0 ; j+\frac{1}{2}, j+\frac{1}{2}, j_{3}-\frac{1}{2}\right\rangle\right. \\
+ & \left.\sqrt{j+j_{3}+1} \bar{Q}^{2}\left|n ; 0 ; j+\frac{1}{2}, j+\frac{1}{2}, j_{3}+\frac{1}{2}\right\rangle\right], \quad j=\frac{|\kappa|-1}{2}, \frac{|\kappa|+1}{2}, \ldots, n-\frac{|\kappa|+3}{2} .
\end{aligned}
$$

\footnotetext{
5 The notation here should not be confused with that introduced at the beginning of Section $\mathrm{V}$, where fermion occupation numbers were used to label states. Here, $\sigma_{1}$ and $\sigma_{2}$ label the supersymmetry multiplet.
} 


\section{Marginally Bound States}

When $2 m E=\theta^{2}$, the algebra is the contraction

$$
\left[\tilde{J}^{i}, \tilde{J}^{j}\right]=i \epsilon^{i j k} \tilde{J}^{k}, \quad\left[\tilde{J}^{i}, \tilde{K}^{j}\right]=i \epsilon^{i j k} \tilde{K}^{k}, \quad\left[\tilde{K}^{i}, \tilde{K}^{j}\right]=0, \quad i=1,2,3 .
$$

Irreducible representations are characterized by the Casimirs (ㅍI.18)

$$
\overrightarrow{\tilde{K}}^{2}=\frac{\kappa^{2} \theta^{2}}{4}, \quad \overrightarrow{\vec{J}} \cdot \overrightarrow{\tilde{K}}=\frac{\kappa^{2} \theta}{4},
$$

and since $\overrightarrow{\tilde{J}}$ and $\overrightarrow{\tilde{K}}$ are hermitian, it is unitary representations that are the representations of interest.

Because of the $\mathrm{SU}(2)$ subalgebra generated by $\overrightarrow{\tilde{J}}$, a general irreducible unitary representation of the algebra (V.24) consists of a sum of SU(2) representations. As reviewed in Appendix C, because $\overrightarrow{\vec{J}} \cdot \overrightarrow{\tilde{K}} \neq 0$, a unitary irreducible representation of the algebra (V.24) contains exactly one copy of every $\mathrm{SU}(2)$ representation $j=j_{0}, j_{0}+1, \ldots$, where $j_{0}=\frac{\overrightarrow{\tilde{J}} \cdot \overrightarrow{\tilde{K}}}{|\tilde{\tilde{K}}|}$.

Thus, for the case at hand, marginally bound states consist of the states with $\overrightarrow{\widetilde{J}}^{2}=\tilde{\jmath}(\tilde{\jmath}+1)$, $\tilde{\jmath}=\frac{|\kappa|}{2}, \frac{|\kappa|}{2}+1, \ldots$ The same argument as in $\oint \mathrm{VB} 1$ then gives the states

$$
\begin{array}{r}
\left|E=\frac{\theta^{2}}{2 m} ; 0 ; j, j, j_{3}\right\rangle, \quad\left|E=\frac{\theta^{2}}{2 m} ; 2 ; j, j, j_{3}\right\rangle=\frac{m}{\theta^{2}} \bar{Q}^{1} \bar{Q}^{2}\left|E=\frac{\theta^{2}}{2 m} ; 0 ; j, j, j_{3}\right\rangle, \quad j=\frac{|\kappa|}{2}, \frac{|\kappa|}{2}+1, \ldots, \\
\begin{aligned}
&\left|E=\frac{\theta^{2}}{2 m} ; 1 ; j-\frac{1}{2}, j, j_{3}\right\rangle=\sqrt{\frac{m}{2 j \theta^{2}}}\left[\sqrt{j+j_{3}} \bar{Q}^{1}\left|E=\frac{\theta^{2}}{2 m} ; 0 ; j-\frac{1}{2}, j-\frac{1}{2}, j_{3}-\frac{1}{2}\right\rangle\right. \\
&\left.+\sqrt{j-j_{3}} \bar{Q}^{2}\left|E=\frac{\theta^{2}}{2 m} ; 0 ; j-\frac{1}{2}, j-\frac{1}{2}, j_{3}+\frac{1}{2}\right\rangle\right], \quad j=\frac{|\kappa|+1}{2}, \frac{|\kappa|+3}{2}, \ldots, \\
& \text { (V.26b) }
\end{aligned} \\
\begin{aligned}
\left|E=\frac{\theta^{2}}{2 m} ; 1 ; j+\frac{1}{2}, j, j_{3}\right\rangle=\sqrt{\frac{m}{2(j+1) \theta^{2}}}\left[-\sqrt{j-j_{3}+1} \bar{Q}^{1}\left|E=\frac{\theta^{2}}{2 m} ; 0 ; j+\frac{1}{2}, j+\frac{1}{2}, j_{3}-\frac{1}{2}\right\rangle\right. \\
\left.+\sqrt{j+j_{3}+1} \bar{Q}^{2}\left|E=\frac{\theta^{2}}{2 m} ; 0 ; j+\frac{1}{2}, j+\frac{1}{2}, j_{3}+\frac{1}{2}\right\rangle\right], \quad j=\frac{|\kappa|-1}{2}, \frac{|\kappa|+1}{2}, \ldots,
\end{aligned}
\end{array}
$$

for the marginally bound states in an angular momentum basis.

\section{Unbound States}

When $2 m E>\theta^{2}$, the relevant algebra is $\mathrm{SO}(3,1)$,

$$
\begin{gathered}
{\left[\tilde{J}^{i}, \tilde{J}^{j}\right]=i \epsilon^{i j k} \tilde{J}^{k}, \quad\left[\tilde{J}^{i}, \hat{K}^{j}\right]=i \epsilon^{i j k} \hat{K}^{k}, \quad\left[\hat{K}^{i}, \hat{K}^{j}\right]=-i \epsilon^{i j k} \tilde{J}^{k}, \quad i=1,2,3,} \\
\overrightarrow{\hat{K}} \equiv \frac{1}{\sqrt{2 m E-\theta^{2}}} \overrightarrow{\tilde{K}} .
\end{gathered}
$$


Irreducible representations are characterized by the Casimirs (ㅍI.18)

$$
\overrightarrow{\tilde{J}}^{2}-\overrightarrow{\hat{K}}^{2}=\frac{\kappa^{2}}{2}-1-\frac{\kappa^{2}}{2} \frac{m E}{2 m E-\theta^{2}}, \quad \quad \overrightarrow{\tilde{J}} \cdot \overrightarrow{\hat{K}}=\frac{\kappa^{2} \theta}{4 \sqrt{2 m E-\theta^{2}}},
$$

and since $\overrightarrow{\tilde{J}}$ and $\overrightarrow{\hat{K}}$ are hermitian for $2 m E>\theta^{2}$, it is unitary representations that are the representations of interest.

Because of the $\mathrm{SU}(2)$ subalgebra generated by $\overrightarrow{\tilde{J}}$, a general irreducible unitary representation of the algebra (V.24) consists of a sum of $\mathrm{SU}(2)$ representations. According to the $\mathrm{SO}(3,1)$ representation theory reviewed in Appendix C, the Casimirs of the relevant unitary irreducible representation are $\overrightarrow{\vec{J}} \cdot \overrightarrow{\hat{K}}=\tilde{\jmath}_{0} \zeta$ and $\overrightarrow{\tilde{J}}^{2}-\overrightarrow{\hat{K}}^{2}=\zeta^{2}+1-\tilde{\jmath}_{0}^{2}$, where $\tilde{\jmath}_{0}$ is a half-integer which labels the minimal irreducible $\mathrm{SU}(2)$ representation in the irreducible representation of the full algebra (V.24) and $\zeta$ is real. The irreducible representation contains exactly one copy of every $\mathrm{SU}(2)$ representation with $\tilde{\jmath}=\tilde{\jmath}_{0}, \tilde{\jmath}_{0}+1, \ldots$ In the case at hand, $\tilde{\jmath}_{0}=\frac{|\kappa|}{2}$, exactly as in the marginally bound case $\oint \mathrm{VC}$, with the same conclusions.

\section{WAVEFUNCTIONS}

Now begins the task of constructing wavefunctions. Roughly speaking, the wavefunctions can be separated into a radial part and an angular part. This is strictly true for the bosonic states - those with even fermion number - and it is only slightly more complicated for the fermionic states with fermion number one. Moreover, the fermionic states are found from the bosonic states using Eqs. (V.23) or ( $(\mathrm{V} .26)$.

\section{A. Angular Momentum Eigenfunctions}

Because the bosonic part of the Hamiltonian differs from that of a charged particle in a magnetic monopole background only by radial terms, the angular dependence of the bosonic states is given by the spherical harmonics for a particle in a magnetic monopole background. In polar coordinates $\vec{x}=(r \sin \vartheta \cos \phi, r \sin \vartheta \sin \phi, r \cos \vartheta)$, and restricting to the northern hemisphere $\left(\vartheta<\frac{\pi}{2}+\epsilon\right)$, these are [26],

$$
\begin{array}{r}
Y_{j, j_{3}}(\vartheta, \phi)=\frac{(-1)^{j-j_{3}}}{2^{j_{3}} \sqrt{4 \pi}} \sqrt{\frac{(2 j+1)\left(j+j_{3}\right) !\left(j-j_{3}\right) !}{\left(j+\frac{\kappa}{2}\right) !\left(j-\frac{\kappa}{2}\right) !}} P_{j-j_{3}}^{\left(j_{3}-\frac{\kappa}{2}, j_{3}+\frac{\kappa}{2}\right)}(\cos \vartheta) \sin ^{j_{3}} \vartheta \cot ^{\frac{\kappa}{2}} \frac{\vartheta}{2} e^{i\left(j_{3}-\frac{\kappa}{2}\right) \phi}, \\
\vartheta<\frac{\pi}{2}+\epsilon, \quad j_{3}=-j,-j+1, \ldots, j-1, j, \quad \text { (VI.1) }
\end{array}
$$


where $^{6}$

$$
P_{n}^{(\alpha, \beta)}(x)=\frac{\Gamma(n+\alpha+1)}{n ! \Gamma(\alpha+1)}{ }_{2} F_{1}\left(-n, \alpha+\beta+n+1 ; \alpha+1 ; \frac{1-x}{2}\right)
$$

is the Jacobi polynomial, expressed as a hypergeometric function. Notice that singlevaluedness (in $\phi$ ) requires $j-\frac{\kappa}{2} \in \mathbb{Z}$; normalizability then demands the bound $j \geq \frac{|\kappa|}{2}$, which was observed in the bosonic theory both classically [Eq. (IV.3)] and quantum mechanically [Eqs. (V.22) and (V.26a $)]$.

In the southern hemisphere $\left(\vartheta>\frac{\pi}{2}-\epsilon\right)$ the gauge transformation (II.3) yields

$$
\begin{array}{r}
Y_{j, j_{3}}(\vartheta, \phi)=\frac{(-1)^{j-j_{3}}}{2^{j_{3}} \sqrt{4 \pi}} \sqrt{\frac{(2 j+1)\left(j+j_{3}\right) !\left(j-j_{3}\right) !}{\left(j+\frac{\kappa}{2}\right) !\left(j-\frac{\kappa}{2}\right) !}} P_{j-j_{3}}^{\left(j_{3}-\frac{\kappa}{2}, j_{3}+\frac{\kappa}{2}\right)}(\cos \vartheta) \sin ^{j_{3}} \vartheta \cot ^{\frac{\kappa}{2}} \frac{\vartheta}{2} e^{i\left(j_{3}+\frac{\kappa}{2}\right) \phi}, \\
\vartheta>\frac{\pi}{2}-\epsilon, \quad j_{3}=-j,-j+1, \ldots, j-1, j . \quad \text { (VI.3) }
\end{array}
$$

Because the only difference is in the sign in front of $\kappa$ in the phase $e^{ \pm i \frac{\kappa}{2} \phi}$, we will, without further comment, take $\epsilon$ only slightly less than $\frac{\pi}{2}$ so that essentially only those points on the negative $z$ axis are not covered by the patch in the upper "hemi" sphere. That is, we will be content to use (VI.1) for the angular dependence, with the understanding that near the south pole, (VI.3) should be used instead.

The spherical harmonics (VI.1) are easily verified by using the identities (VI.2) and $\frac{d}{d x} 2 F_{1}(\alpha, \beta ; \gamma ; x)=\frac{\alpha \beta}{\gamma}{ }_{2} F_{1}(\alpha, \beta ; \gamma ; x)$ to find

$$
\frac{d}{d x} P_{n}^{(\alpha, \beta)}(x)=\frac{1}{2}(\alpha+\beta+n+1) P_{n-1}^{(\alpha+1, \beta+1)}(x) .
$$

It is then straightforward to verify

$$
J^{+} Y_{j, j_{3}}(\vartheta, \phi)=\sqrt{\left(j-j_{3}\right)\left(j+j_{3}+1\right)} Y_{j, j_{3}+1}(\vartheta, \phi), \quad J^{3} Y_{j, j_{3}}(\vartheta, \phi)=j_{3} Y_{j, j_{3}}(\vartheta, \phi) .
$$

With a little more work ${ }^{7}$ one can also verify

$$
J^{-} Y_{j, j_{3}}(\vartheta, \phi)=\sqrt{\left(j+j_{3}\right)\left(j-j_{3}+1\right)} Y_{j, j_{3}-1}(\vartheta, \phi) .
$$

It is easily seen that $Y_{j, j}(\vartheta, \phi)$ is properly normalized; the recursion then ensures that $Y_{j, j_{3}}(\vartheta, \phi)$ is.

The bosonic harmonics (VI.1) yield fermionic harmonics upon adding spin via the

\footnotetext{
${ }^{6}$ It is understood here that $\frac{1}{\Gamma(c)}{ }_{2} F_{1}(-n, b ; c ; z) \equiv \frac{n !}{\Gamma(b)} \sum_{p=0}^{n}(-1)^{p} \frac{\Gamma(b+p)}{p !(n-p) ! \Gamma(c+p)} z^{p}$, which is well-defined even when $c$ is a nonpositive integer, provided that $b$ is not also a nonpositive integer. Since $\left(j_{3}-\frac{\kappa}{2}\right)+\left(j_{3}+\frac{\kappa}{2}\right)+\left(j-j_{3}\right)+1=j+j_{3}+1 \geq 1$, this condition on $b$ is satisfied in this paper.

7 It may be useful to use Eq. (B.4b).
} 
Clebsch-Gordan coefficients. This results in

$$
\begin{aligned}
& \left\langle\vartheta, \phi \mid j-\frac{1}{2}, j, j_{3}\right\rangle=\sqrt{\frac{j+j_{3}}{2 j}} Y_{j-\frac{1}{2}, j_{3}-\frac{1}{2}}(\vartheta, \phi)|10\rangle+\sqrt{\frac{j-j_{3}}{2 j}} Y_{j-\frac{1}{2}, j_{3}+\frac{1}{2}}(\vartheta, \phi)|01\rangle, \quad \text { (VI.6a) } \\
& \left\langle\vartheta, \phi \mid j+\frac{1}{2}, j, j_{3}\right\rangle=-\sqrt{\frac{j-j_{3}+1}{2(j+1)}} Y_{j+\frac{1}{2}, j_{3}-\frac{1}{2}}(\vartheta, \phi)|10\rangle+\sqrt{\frac{j+j_{3}+1}{2(j+1)}} Y_{j+\frac{1}{2}, j_{3}+\frac{1}{2}}(\vartheta, \phi)|01\rangle,
\end{aligned}
$$

using the basis for fermionic states, Eq. (V.1). These states are single-valued if $j$ differs from $\frac{\kappa}{2}$ by half an odd integer. In particular, the states (VI.6a) are well-defined for $0 \leq j-\frac{\kappa+1}{2} \in \mathbb{Z}$ and the states (VI.6b) are well-defined for $0 \leq j-\frac{\kappa-1}{2} \in \mathbb{Z}$.

Please do not be fooled by the resemblance between the notation in the definitions (VI.6) and (V.23). The states (V.23) have a definite $\overrightarrow{\widetilde{J}}^{2}$ and are otherwise defined by the angular

momentum $\vec{J}=\overrightarrow{\tilde{J}}+\vec{S}$, but for the harmonics (VI.6), the angular momentum has been decomposed as a sum of orbital and spin angular momenta, ie. $\vec{J}=\vec{L}+\vec{s}$. The former are natural from a supersymmetric perspective, but the latter is more physical.

\section{B. Ground State Wavefunctions}

Because of the SU $(2)^{2}$ symmetry algebra, generated by $\vec{N}_{ \pm}$[Eq. (V.4)], associated with the ground states, one ground state can be found by demanding that it is annihilated by the raising operators $N_{ \pm}^{+}$, and that it be an eigenstate of $N_{ \pm}^{3}$. The remaining ground states are found by applying the lowering operators $N_{ \pm}^{-}$.

In fact, because of the Casimirs (V.10), the equations to solve are equivalently

$$
\begin{aligned}
J^{+}\left|E ; \frac{|\kappa|-1}{2}, \frac{|\kappa|-1}{2}\right\rangle & =0, & J^{3}\left|E ; \frac{|\kappa|-1}{2}, \frac{|\kappa|-1}{2}\right\rangle & =\frac{|\kappa|-1}{2}\left|E ; \frac{|\kappa|-1}{2}, \frac{|\kappa|-1}{2}\right\rangle, \\
K^{+}\left|E ; \frac{|\kappa|-1}{2}, \frac{|\kappa|-1}{2}\right\rangle & =0, & K^{3}\left|E ; \frac{|\kappa|-1}{2}, \frac{|\kappa|-1}{2}\right\rangle & =\frac{|\kappa|-1}{2}\left|E ; \frac{|\kappa|-1}{2}, \frac{|\kappa|-1}{2}\right\rangle
\end{aligned}
$$

and moreover it was learned in $8 \mathrm{VA}$ that these states have fermion number one. But Eqs. (VI.7a) have already been solved, more generally, by Eq.(VI.6b). That is, in a coordinate basis, the ground states are given by

$$
\left\langle r, \vartheta, \phi \mid E ; \frac{|\kappa|-1}{2}, j_{3}\right\rangle=-\sqrt{\frac{|\kappa|+1-2 j_{3}}{2(|\kappa|+1)}} R_{g}(r) Y_{\frac{|\kappa|}{2}, j_{3}-\frac{1}{2}}(\vartheta, \phi)|10\rangle+\sqrt{\frac{|\kappa|+1+2 j_{3}}{2(|\kappa|+1)}} R_{g}(r) Y_{\frac{|\kappa|}{2}, j_{3}+\frac{1}{2}}(\vartheta, \phi)|01\rangle .
$$

In particular, the state annihilated by $J^{+}$and $K^{+}$is

$$
\begin{aligned}
\left\langle r, \vartheta, \phi \mid E ; \frac{|\kappa|-1}{2}, \frac{|\kappa|-1}{2}\right\rangle=\sqrt{\frac{|\kappa|}{4 \pi}} R_{g}(r) \sin \frac{|\kappa|-\kappa}{2} \frac{\vartheta}{2} \cos \frac{|\kappa|+\kappa}{2} \frac{\vartheta}{2} e^{i \frac{|\kappa|-\kappa}{2} \phi} \\
\times\left[\left(1-\frac{\kappa}{|\kappa|}-2 \sin ^{2} \frac{\vartheta}{2}\right) \csc \vartheta e^{-i \phi}|10\rangle+|01\rangle\right] .
\end{aligned}
$$

It remains to determine the radial function $R_{g}(r)$. 
The radial function is determined by solving $K^{+}\left|E ; \frac{|\kappa|-1}{2}, \frac{|\kappa|-1}{2}\right\rangle=0$. A little algebra converts the component of this equation along $|01\rangle$ into

$$
R_{g}^{\prime}(r)=\frac{1}{r}\left(\frac{|\kappa|}{2}-1\right) R_{g}(r)+\frac{\kappa \theta}{|\kappa|} R_{g}(r),
$$

and therefore

$$
R_{g}(r)=\frac{(2|\theta|)^{3 / 2}}{\sqrt{(|\kappa|) !}}(2|\theta| r)^{\frac{|\kappa|}{2}-1} e^{\frac{\kappa}{|\kappa|} \theta r} .
$$

Notice that this is normalizable only if $\kappa \theta<0$, the same condition found for the existence of classically bound orbits.

Applying the lowering operator $J^{-}$thus yields the ground state wavefunctions

$$
\begin{aligned}
&\langle r, \vartheta, \phi| 0 ;\left.\frac{|\kappa|-1}{2}, j_{3}\right\rangle=(-1)^{\frac{|\kappa|-1}{2}-j_{3}} \frac{2|\theta|^{3 / 2}}{\sqrt{\pi}|\kappa| !} \sqrt{\left(\frac{|\kappa|-1}{2}+j_{3}\right) !\left(\frac{|\kappa|-1}{2}-j_{3}\right) !} \\
& \times(2|\theta| r)^{\frac{|\kappa|}{2}-1} e^{\frac{\kappa}{|\kappa|} \theta r} \sin ^{j_{3}-\frac{1}{2}} \vartheta \cot ^{\frac{\kappa}{2}} \frac{\vartheta}{2} e^{i \frac{|\kappa|-\kappa}{2} \phi} \\
& \times\left[P_{\frac{|\kappa|+\frac{1}{2}}{2}-j_{3}}^{\left(j_{3}-\frac{\kappa+1}{2}, j_{3}+\frac{\kappa-1}{2}\right)}(\cos \vartheta) e^{-i \phi}|10\rangle+\frac{1}{2} \sin \vartheta P_{\frac{|\kappa|-1}{2}-j_{3}}^{\left(j_{3}-\frac{\kappa-1}{2}, j_{3}+\frac{\kappa+1}{2}\right)}(\cos \vartheta)|01\rangle\right] .
\end{aligned}
$$

Again, these states only exist if $\kappa \theta<0$ and for $j_{3}=-\frac{|\kappa|-1}{2}, \ldots, \frac{|\kappa|-1}{2}$. There are $|\kappa|$ of these states.

\section{Bound State Wavefunctions}

In this section, we present the wavefunctions of the bound states. Recall from $\$ \mathrm{VB}$ that the algebra of $\overrightarrow{\tilde{N}}_{ \pm}$gives the states $\left|n ; \sigma_{1}, \sigma_{2} ; n_{3}^{-}, n_{3}^{+}\right\rangle$. If these were the states of interest, then operator techniques such as those in $\S \mathrm{VIB}$ would give the wavefunctions. In particular, $\left|n ; \sigma_{1}, \sigma_{2} ; \frac{n-1}{2}, \frac{n-|\kappa|-1}{2}\right\rangle$ is found by demanding that it be annihilated by $\tilde{K}^{+}$and $\tilde{J}^{+}$, and that it have the correct $\tilde{K}_{3}$ and $\tilde{J}_{3}$ eigenvalues.

However, the relation of those states to the states of $\S \mathrm{VB} 1,\left|n ; \sigma_{1}+\sigma_{2} ; \tilde{\jmath}, j, j_{3}\right\rangle$, is sufficiently nontrivial, that a more brute force method will be applied. We will use our knowledge of the angular part of the wavefunction to find the equation of motion for the radial factor directly from the Hamiltonian. Fermionic wavefunctions are then found using Eq. (V.23).

\section{Bosonic Bound States}

The wavefunctions of bosonic bound states will have the form

$$
\langle\sigma \sigma|\left\langle r, \vartheta, \phi \mid n ; 2 \sigma ; j, j, j_{3}\right\rangle=R_{n, j}(r) Y_{j, j_{3}}(\vartheta, \phi), \quad \sigma=0,1, \quad \begin{aligned}
& j=\frac{|\kappa|}{2}, \ldots, n-\frac{|\kappa|}{2}-1 \\
& j_{3}=-j, \ldots, j .
\end{aligned}
$$


Given the energy eigenvalue (V.17), and writing the Hamiltonian (III.2) in terms of $\vec{J}^{2}$ then yields the equation

$$
-R_{n, j}^{\prime \prime}(r)-\frac{2}{r} R_{n, j}^{\prime}(r)+\frac{j(j+1)}{r^{2}} R_{n, j}(r)+\frac{\kappa \theta}{r} R_{n, j}+\theta^{2} R_{n, j}(r)=\frac{4 n \theta^{2}(n-|\kappa|)}{(2 n-|\kappa|)^{2}} R_{n, j}(r) .
$$

Notice that the $\frac{\kappa^{2}}{8 m r^{2}}$ part of the potential was absorbed into $\frac{\vec{J}^{2}}{2 m r^{2}}$ (and we have multiplied Schrödinger's equation by $2 m$ ). If $\kappa \theta>0$, there are no regular solutions, as expected. ${ }^{8}$ Otherwise, for $\kappa \theta<0$, the regular solution is given in terms of an associated Laguerre polynomial, $L_{n}^{k}(x)$,

$$
R_{n, j}(r)=\frac{(-2 \theta \kappa)^{3 / 2}}{(2 n-|\kappa|)^{2}} \sqrt{\frac{\left(n-j-\frac{|\kappa|}{2}-1\right) !}{\left(n+j-\frac{|\kappa|}{2}\right) !}}\left(-\frac{2 \theta \kappa}{2 n-|\kappa|} r\right)^{j} L_{n-j-\frac{|\kappa|}{2}-1}^{2 j+1}\left(-\frac{2 \theta \kappa}{2 n-|\kappa|} r\right) e^{\frac{\theta \kappa}{2 n-|\kappa|} r}
$$

Thus, the bound (but excited) state bosonic wavefunctions are

$$
\begin{aligned}
& \langle\sigma \sigma|\left\langle r, \vartheta, \phi \mid n ; 2 \sigma ; j, j, j_{3}\right\rangle=\frac{(-1)^{j-j_{3}}(-2 \theta \kappa)^{3 / 2}}{2^{j_{3}}(2 n-|\kappa|)^{2} \sqrt{4 \pi}} \sqrt{\frac{(2 j+1)\left(n-j-\frac{|\kappa|}{2}-1\right) !\left(j+j_{3}\right) !\left(j-j_{3}\right) !}{\left(n+j-\frac{|\kappa|}{2}\right) !\left(j+\frac{\kappa}{2}\right) !\left(j-\frac{\kappa}{2}\right) !}} \\
& \times\left(-\frac{2 \theta \kappa}{2 n-|\kappa|} r\right)^{j} L_{n-j-\frac{|\kappa|}{2}-1}^{2 j+1}\left(-\frac{2 \theta \kappa}{2 n-|\kappa|} r\right) e^{\frac{\theta \kappa}{2 n-|\kappa|}} P_{j-j_{3}}^{\left(j-\frac{\kappa}{2}, j_{3}+\frac{\kappa}{2}\right)}(\cos \vartheta) \sin ^{j_{3}} \vartheta \cot ^{\frac{\kappa}{2}} \frac{\vartheta}{2} e^{i\left(j_{3}-\frac{\kappa}{2}\right) \phi} .
\end{aligned}
$$

This result might have been partially anticipated if one reasoned that since the classical trajectories are conic sections, one should get radial dependence similar to the hydrogen atom; however, it is not obvious that this should be true since the plane of classical trajectories is off of the origin.

\section{Fermionic Bound States}

Fermionic states are found from the bosonic ones, Eq. (VI.17) with $\sigma=0$, using Eq. (V.23)..$^{9}$ By matching powers of $x$ it is possible to demonstrate that

$$
L_{n}^{\prime(\alpha)}(x)=-\frac{n}{\alpha+1} L_{n}^{(\alpha)}(x)-\frac{x}{\alpha+1} L_{n-1}^{(\alpha+2)}(x),
$$

${ }^{8}$ For $\kappa \theta>0$, the putative solution would be

$$
R_{n, j}(r) \propto r^{j} L_{-n-j+\frac{|\kappa|}{2}-1}^{2 j+1}\left(\frac{2 \theta \kappa}{2 n-|\kappa|}\right) e^{-\frac{\theta \kappa}{2 n-|\kappa|} r} .
$$

However, the index of the Laguerre polynomial is negative. Thus, either this expression is zero, or the proportionality constant is infinite, whereupon the "polynomial" is an infinite series whose asymptotic behaviour is $e^{\frac{2 \theta \kappa}{2 n-|\kappa|} r}$ and so leads to a divergent function.

9 See Appendix B. 
and therefore

$$
R_{n, j}^{\prime}(r)=\left(\frac{j}{r}+\frac{\theta \kappa}{2(j+1)}\right) R_{n, j}(r)+\frac{\theta \kappa}{2 n-|\kappa|} \sqrt{\frac{(2 n-|\kappa|)^{2}}{4(j+1)^{2}}-1} R_{n, j+1}(r) .
$$

Thus, using Eq. (B.7) one can write the bound state fermionic wavefunctions in the form

$$
\begin{aligned}
& \left\langle r, \vartheta, \phi \mid n ; 1 ; j-\frac{1}{2}, j, j_{3}\right\rangle=\frac{1}{\sqrt{n(n-|\kappa|)}}\left\{\frac{\frac{\kappa(2 n-|\kappa|)}{2 j+1} R_{n, j-\frac{1}{2}}(r)+\kappa \sqrt{\left(\frac{2 n-|\kappa|}{2 j+1}\right)^{2}-1} R_{n, j+\frac{1}{2}}(r)}{2 j+1} \sqrt{\left(j+\frac{1}{2}\right)^{2}-\frac{\kappa^{2}}{4}}\left\langle\vartheta, \phi \mid j+\frac{1}{2}, j, j_{3}\right\rangle\right. \\
& \left.+\left(\kappa \frac{\frac{\kappa(2 n-|\kappa|)}{2 j+1} R_{n, j-\frac{1}{2}}(r)+\kappa \sqrt{\left(\frac{2 n-|\kappa|}{2 j+1}\right)^{2}-1} R_{n, j+\frac{1}{2}}(r)}{2(2 j+1)}-\frac{2 n-|\kappa|}{2} R_{n, j-\frac{1}{2}}(r)\right)\left\langle\vartheta, \phi \mid j-\frac{1}{2}, j, j 3\right\rangle\right\}, \\
& \left\langle r, \vartheta, \phi \mid n ; 1 ; j+\frac{1}{2}, j, j_{3}\right\rangle=\frac{1}{\sqrt{n(n-|\kappa|)}}\{ \\
& \quad \frac{\left(\frac{2(j+1)}{\theta r}+\frac{\kappa}{2 j+3}\right)(2 n-|\kappa|) R_{n, j+\frac{1}{2}}(r)+\kappa \sqrt{\left(\frac{2 n-|\kappa|}{2 j+3}\right)^{2}-1} R_{n, j+\frac{3}{2}}(r)}{2 j+1} \sqrt{\left(j+\frac{1}{2}\right)^{2}-\frac{\kappa^{2}}{4}}\left\langle\vartheta, \phi \mid j-\frac{1}{2}, j, j_{3}\right\rangle \\
& \left.-\left(\kappa \frac{\left(\frac{2(j+1)}{\theta r}+\frac{\kappa}{2 j+3}\right)(2 n-|\kappa|) R_{n, j+\frac{1}{2}}(r)+\kappa \sqrt{\left(\frac{2 n-|\kappa|}{2 j+3}\right)^{2}-1} R_{n, j+\frac{3}{2}}(r)}{2(2 j+1)}+\frac{2 n-|\kappa|}{2} R_{n, j+\frac{1}{2}}(r)\right)\left\langle\vartheta, \phi \mid j+\frac{1}{2}, j, j 3\right\rangle\right\} .
\end{aligned}
$$

\section{Unbound States}

\section{Bosonic Unbound States}

Replacing the eigenvalue on the right hand side of the radial equation (VI.14) with $2 m E-\theta^{2}$ immediately leads to the radial eigenfunctions

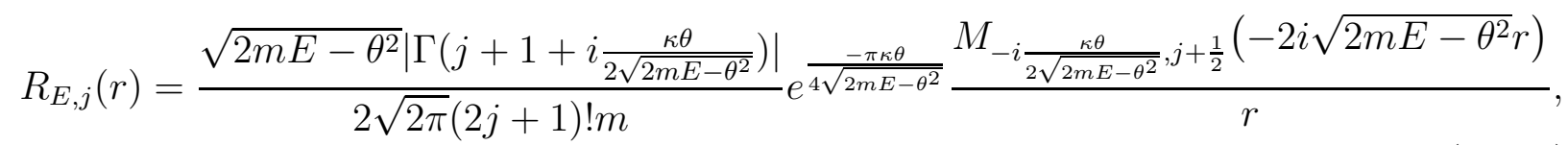

where $M_{k, \mu}(x)$ is the Whittaker function,

$$
M_{k, \mu}(x)=e^{-x / 2} x^{\mu+\frac{1}{2}}{ }_{1} F_{1}\left(\mu-k+\frac{1}{2} ; 2 \mu+1 ; x\right) .
$$

The confluent hypergeometric function which appears might also be called a nonpolynomial Laguerre function.

In the wavefunction (VI.21) a particular square root of $\left(2 m E-\theta^{2}\right)$ has been chosen; the identity [28, Eq. (9.231.2)] shows that the wavefunction with the other square root differs from this one by at most a sign. The normalization has been chosen so that

$$
\int_{0}^{\infty} d r r^{2} R_{E^{\prime}, j}^{*}(r) R_{E, j}(r)=\delta\left(E-E^{\prime}\right),
$$

thus ensuring orthonormal wavefunctions. See appendix D. 


\section{Fermionic Unbound States}

Finding the fermionic unbound state wavefunctions is a simple matter of applying Eq. (B.7) for the radial dependence in Eq. (VI.21). Using the identity

$$
M_{k, \mu}^{\prime}(x)=\frac{1}{x}\left(\mu+\frac{1}{2}\right) M_{k, \mu}(x)-\frac{k}{2 \mu+1} M_{k, \mu}(x)+\frac{\left(\mu+\frac{1}{2}\right)^{2}-k^{2}}{2(2 \mu+1)^{2}(\mu+1)} M_{k, \mu+1}(x),
$$

which can be derived from the confluent hypergeometric representation, it is possible to write

$$
R_{E, j}^{\prime}(r)=\left(\frac{j}{r}-\frac{\kappa \theta}{2(j+1)}\right) R_{E, j}(r)-i \sqrt{2 m E-\theta^{2}+\frac{\kappa^{2} \theta^{2}}{4(j+1)^{2}}} R_{E, j+1}(r) .
$$

Plugging into Eq. (B.7), one finds the fermionic wavefunctions in terms of the bosonic radial dependence and the fermionic harmonics

$$
\begin{aligned}
& \left\langle r, \vartheta, \phi \mid E ; 1 ; j-\frac{1}{2}, j, j_{3}\right\rangle=-\frac{1}{\sqrt{2 m E}}\left\{\frac{i \sqrt{2 m E-\theta^{2}+\frac{\kappa^{2} \theta^{2}}{(2 j+1)^{2}}} R_{E, j+\frac{1}{2}}(r)+\frac{\kappa \theta}{2 j+1} R_{E, j-\frac{1}{2}}(r)}{j+1} \sqrt{\left(j+\frac{1}{2}\right)^{2}-\frac{\kappa^{2}}{4}}\left\langle\vartheta, \phi \mid j+\frac{1}{2}, j, j_{3}\right\rangle\right. \\
& \left.+\left(\kappa \frac{i \sqrt{2 m E-\theta^{2}+\frac{\kappa^{2} \theta^{2}}{(2 j+1)^{2}}} R_{E, j+\frac{1}{2}}(r)+\frac{\kappa \theta}{2 j+1} R_{E, j-\frac{1}{2}}(r)}{2(j+1)}+\theta R_{E, j-\frac{1}{2}}(r)\right)\left\langle\vartheta, \phi \mid j-\frac{1}{2}, j, j_{3}\right\rangle\right\}, \\
& \left\langle r, \vartheta, \phi \mid E ; 1 ; j+\frac{1}{2}, j, j_{3}\right\rangle=\frac{1}{\sqrt{2 m E}}\left\{4 \frac{\left(\frac{j+1}{r}-\frac{\kappa \theta}{2 j+3}\right) R_{E, j+\frac{1}{2}}(r)-i \sqrt{2 m E-\theta^{2}+\frac{\kappa^{2} \theta^{2}}{(2 j+3)^{2}}} R_{E, j+\frac{3}{2}}(r)}{2 j+1} \sqrt{\left(j+\frac{1}{2}\right)^{2}-\frac{\kappa^{2}}{4}}\left\langle\vartheta, \phi \mid j-\frac{1}{2}, j, j_{3}\right\rangle\right. \\
& \left.-\left(2 \kappa \frac{\left(\frac{j+1}{r}-\frac{\kappa \theta}{2 j+3}\right) R_{E, j+\frac{1}{2}}(r)-i \sqrt{2 m E-\theta^{2}+\frac{\kappa^{2} \theta^{2}}{(2 j+3)^{2}}} R_{E, j+\frac{3}{2}}(r)}{2 j+1}+\theta R_{E, j+\frac{1}{2}}(r)\right)\left\langle\vartheta, \phi \mid j+\frac{1}{2}, j, j_{3}\right\rangle\right\} .
\end{aligned}
$$

\section{E. Marginally Bound States}

\section{Bosonic States}

The radial dependence for the marginally bound states with $2 m E=\theta^{2}$ is

$$
R_{E=\frac{\theta^{2}}{2 m}, j}(r) \propto \frac{1}{\sqrt{-\kappa \theta r}} J_{2 j+1}(2 \sqrt{-\kappa \theta r})
$$

which is well-behaved at $r=\infty$ if $\kappa$ and $\theta$ have opposite sign. The proper normalization is a $\delta$-function normalization with the unbound states. To be precise, we note that evaluating the radial wavefunction of the unbound states near $2 m E=\theta^{2}$ gives

$$
R_{E \approx \frac{\theta^{2}}{2 m}, j}(r) \approx \frac{\left(2 m E-\theta^{2}\right)^{3 / 4}}{m} \sqrt{\frac{-\kappa \theta}{2}} e^{-\frac{\pi(\kappa \theta+|\kappa \theta|)}{4 \sqrt{2 m E-\theta^{2}}}} \frac{J_{2 j+1}(2 \sqrt{-\kappa \theta r})}{\sqrt{-\kappa \theta r}},
$$

after dropping an irrelevant phase. Notice that the exponential factor is trivial when $\kappa \theta<0$, but forces the threshold wavefunction to strongly vanish for $\kappa \theta>0$, in accord with the presence or absence of bound states. 


\section{Fermionic States}

Applying a standard Bessel function identity [29, Eq. (9.1.27)], one can demonstrate that

$$
R_{E=\frac{\theta^{2}}{2 m}, j}^{\prime}(r)=-\frac{R_{E=\frac{\theta^{2}}{2 m}, j}(r)}{2 r}+\frac{1}{2} \sqrt{-\frac{\kappa \theta}{r}} R_{E=\frac{\theta^{2}}{2 m}, j-\frac{1}{2}}(r)-\frac{1}{2} \sqrt{-\frac{\kappa \theta}{r}} R_{E=\frac{\theta^{2}}{2 m}, j+\frac{1}{2}}(r),
$$

from which one finds the fermionic wave functions in terms of the bosonic radial dependence and the fermionic harmonics using Eq. (B.7):

$$
\begin{aligned}
& \left\langle r, \vartheta, \phi \mid E=\frac{\theta^{2}}{2 m} ; 1 ; j-\frac{1}{2}, j, j_{3}\right\rangle=\frac{1}{\theta}\left\{\frac{\sqrt{\frac{-\kappa \theta}{r}}\left(R_{j-1}(r)-R_{j}(r)\right)-\frac{2 j}{r} R_{j-\frac{1}{2}}(r)}{2 j+1} \sqrt{\left(j+\frac{1}{2}\right)^{2}-\frac{\kappa^{2}}{4}}\left\langle\vartheta, \phi \mid j+\frac{1}{2}, j, j_{3}\right\rangle\right. \\
& \left.+\left(\kappa \frac{\frac{1}{2} \sqrt{\frac{-\kappa \theta}{r}}\left(R_{j-1}(r)-R_{j}(r)\right)-\frac{j}{r} R_{j-\frac{1}{2}}(r)}{2 j+1}-\theta R_{j-\frac{1}{2}}(r)\right)\left\langle\vartheta, \phi \mid j-\frac{1}{2}, j, j_{3}\right\rangle\right\}, \\
& \left\langle r, \vartheta, \phi \mid E=\frac{\theta^{2}}{2 m} ; 1 ; j+\frac{1}{2} \cdot j, j_{3}\right\rangle=\frac{1}{\theta}\left\{\frac{\sqrt{\frac{-\kappa \theta}{r}}\left(R_{j}(r)-R_{j+1}(r)\right)+\frac{2(j+1)}{r} R_{j+\frac{1}{2}}(r)}{2 j+1} \sqrt{\left(j+\frac{1}{2}\right)^{2}-\frac{\kappa^{2}}{4}}\left\langle\vartheta, \phi \mid j-\frac{1}{2}, j, j_{3}\right\rangle\right. \\
& \left.\quad-\left(\kappa \frac{\frac{1}{2} \sqrt{\frac{-\kappa \theta}{r}}\left(R_{j}(r)-R_{j+1}(r)\right)+\frac{j+1}{r} R_{j+\frac{1}{2}}(r)}{2 j+1}+\theta R_{j+\frac{1}{2}}(r)\right)\left\langle\vartheta, \phi \mid j+\frac{1}{2}, j, j_{3}\right\rangle\right\},
\end{aligned}
$$

where, as should be clear, we have dropped the $E=\frac{\theta^{2}}{2 m}$ subscript on all of the above $R$ 's.

\section{Acknowledgments}

We thank B. Chowdhury, S. Mathur and S. Nussinov for useful conversations. We also thank M. Headrick for making his useful and instructive Mathematica ${ }^{\circledR}$ package grassmann.m available. This work was supported in part by Department of Energy contract No. DE-FG02-91ER-40690.

\section{APPENDIX A: CONVENTIONS}

Indexed bosonic coordinates $\vec{x}=\left(x^{1}, x^{2}, x^{3}\right)$ and coordinates $\vec{x}=(x, y, z)$ are used interchangeably. Overdots denote time derivatives: $\dot{\vec{x}} \equiv \frac{d \vec{x}}{d t}$.

Spinor fields $\lambda, \xi$ are two-component spinors whose conjugates are $\bar{\lambda}$ and $\bar{\xi}$. If needed, the spinor indices $\alpha, \beta, \cdots=1,2$ will be such that $\bar{\lambda}^{\alpha}=\left(\lambda_{\alpha}\right)^{*}$, so that $\bar{\lambda} \lambda \equiv \bar{\lambda}^{\alpha} \lambda_{\alpha}$ is a scalar. In this paper, we never need to contract two unbarred or two barred spinors, and so we do not specify a convention for this unnecessary operation.

The standard Pauli matrices are $\sigma^{i}$, or $\sigma_{\alpha}^{i \beta}$. In addition we use the standard raising and lowering operators

$$
\sigma^{ \pm}=\frac{1}{2}\left(\sigma^{1} \pm i \sigma^{2}\right)
$$

so that $\left(\sigma^{+}\right)=\left(\begin{array}{ll}0 & 1 \\ 0 & 0\end{array}\right)=\left(\sigma^{-}\right)^{\top}$. For all other vectors and one-forms, the \pm components do not have a factor of $\frac{1}{2}$. For example,

$$
x^{+}=x^{1}+i x^{2}=x+i y, \quad p_{+}=p_{1}+i p_{2}, \quad A_{-}=A_{1}-i A_{2} .
$$




\section{APPENDIX B: FERMIONIC WAVEFUNCTIONS}

In this section, the general relationship of fermionic wavefunctions to their bosonic superpartner is derived. The linear combinations of the supersymmetry charges given in Eq. (V.23) show how to find the fermionic states from the bosonic states; however, it is not a trivial matter to write the resulting fermionic wavefunctions in terms of the more physically interpretable fermionic harmonics in Eq. (VI.6). In fact, it requires a number of Jacobi polynomial identities or equivalently bosonic harmonic identities.

In general the bosonic wavefunctions may be written in the form

$$
\left\langle r, \vartheta, \phi \mid E ; 0 ; j, j, j_{3}\right\rangle=R_{E, j}(r) Y_{j, j_{3}}(\vartheta, \phi),
$$

where the angular dependence is given in Eq. (VI.1). We begin by applying the position space representations of the supersymmetry charges in the combinations given in Eq. (V.23) to find

$$
\begin{aligned}
& \left\langle r, \vartheta, \phi \mid E ; 1 ; j-\frac{1}{2}, j, j_{3}\right\rangle \\
& =\frac{1}{\sqrt{4 j m E}}\left\{\sqrt { j + j _ { 3 } } \left[\left(\cos \vartheta \frac{R_{-}^{\prime}}{R_{-}}-\frac{\sin \vartheta}{r} \frac{Y_{--}^{\prime}}{Y_{--}}-\left(\frac{\kappa}{2 r}+\theta\right)\right)|10\rangle\right.\right. \\
& \left.+\left(\sin \vartheta \frac{R_{-}^{\prime}}{R_{-}}+\frac{\cos \vartheta}{r} \frac{Y_{--}^{\prime}}{Y_{--}}+\frac{\frac{\kappa}{2} \cos \vartheta-j_{3}+\frac{1}{2}}{r \sin \vartheta}\right) e^{i \phi}|01\rangle\right] R_{-} Y_{--} \\
& +\sqrt{j-j_{3}}\left[-\left(\cos \vartheta \frac{R_{-}^{\prime}}{R_{-}}-\frac{\sin \vartheta}{r} \frac{Y_{-+}^{\prime}}{Y_{-+}}+\left(\frac{\kappa}{2 r}+\theta\right)\right)|01\rangle\right. \\
& \left.\left.+\left(\sin \vartheta \frac{R_{-}^{\prime}}{R_{-}}+\frac{\cos \vartheta}{r} \frac{Y_{-+}^{\prime}}{Y_{-+}}-\frac{\frac{\kappa}{2} \cos \vartheta-j_{3}-\frac{1}{2}}{r \sin \vartheta}\right) e^{-i \phi}|10\rangle\right] R_{-} Y_{-+}\right\}, \\
& \left\langle r, \vartheta, \phi \mid E ; 1 ; j+\frac{1}{2}, j, j_{3}\right\rangle \\
& =\frac{1}{\sqrt{4(j+1) m E}}\left\{-\sqrt{j-j_{3}+1}\left[\left(\cos \vartheta \frac{R_{+}^{\prime}}{R_{+}}-\frac{\sin \vartheta}{r} \frac{Y_{+-}^{\prime}}{Y_{+-}}-\left(\frac{\kappa}{2 r}+\theta\right)\right) \mid\right.\right. \\
& \left.+\left(\sin \vartheta \frac{R_{+}^{\prime}}{R_{+}}+\frac{\cos \vartheta}{r} \frac{Y_{+-}^{\prime}}{Y_{+-}}+\frac{\frac{\kappa}{2} \cos \vartheta-j_{3}+\frac{1}{2}}{r \sin \vartheta}\right) e^{i \phi}|01\rangle\right] R_{+} Y_{+-} \\
& +\sqrt{j+j_{3}+1}\left[-\left(\cos \vartheta \frac{R_{+}^{\prime}}{R_{+}}-\frac{\sin \vartheta}{r} \frac{Y_{++}^{\prime}}{Y_{++}}+\left(\frac{\kappa}{2 r}+\theta\right)\right)|01\rangle\right. \\
& \left.\left.+\left(\sin \vartheta \frac{R_{+}^{\prime}}{R_{+}}+\frac{\cos \vartheta}{r} \frac{Y_{++}^{\prime}}{Y_{++}}-\frac{\frac{\kappa}{2} \cos \vartheta-j_{3}-\frac{1}{2}}{r \sin \vartheta}\right) e^{-i \phi}|10\rangle\right] R_{+} Y_{++}\right\},
\end{aligned}
$$

with a shorthand

$$
R_{ \pm}=R_{E, j \pm \frac{1}{2}}, \quad Y_{ \pm \pm^{\prime}}=Y_{j \pm \frac{1}{2}, j_{3} \pm^{\prime} \frac{1}{2}}
$$

and where prime denotes differentiation with respect to $r$ or $\vartheta$, depending on the context. To simplify these expressions we need to relate $\partial_{\vartheta} Y_{j, j_{3}}$ to other monopole harmonics. Fortunately, this is not difficult with the identity Eq. (VI.4). Once the expression is entirely in 
terms of the bosonic harmonics there is a sizeable amount of algebraic manipulation required to put the fermionic wavefunctions in terms of the fermionic harmonics in Eq. (VI.6). The relevant Jacobi polynomial identities needed to effect this transformation are

$$
\begin{aligned}
& \frac{d}{d x} P_{n}^{(\alpha, \beta)}(x)=\frac{1}{2}(\alpha+\beta+n+1) P_{n-1}^{(\alpha+1, \beta+1)}(x), \\
& -(n+1) P_{n+1}^{(\alpha-1, \beta-1)}(x)=\frac{1}{4}(n+\alpha+\beta+1)\left(1-x^{2}\right) P_{n-1}^{(\alpha+1, \beta+1)}(x) \\
& +\frac{1}{2}(\beta-\alpha-(\alpha+\beta) x) P_{n}^{(\alpha, \beta)}(x), \\
& x P_{n}^{(\alpha, \beta)}(x)=\frac{2(n+1)(n+\alpha+\beta+1)}{(2 n+\alpha+\beta+1)(2 n+\alpha+\beta+2)} P_{n+1}^{(\alpha, \beta)}(x) \\
& +\frac{\beta^{2}-\alpha^{2}}{(2 n+\alpha+\beta)(2 n+\alpha+\beta+2)} P_{n}^{(\alpha, \beta)}(x) \\
& +\frac{2(n+\alpha)(n+\beta)}{(2 n+\alpha+\beta)(2 n+\alpha+\beta+1)} P_{n-1}^{(\alpha, \beta)}(x), \\
& \left(1-x^{2}\right) P_{n}^{(\alpha, \beta)}(x)=\frac{4(n+\alpha)(n+\beta)}{(2 n+\alpha+\beta)(2 n+\alpha+\beta+1)} P_{n}^{(\alpha-1, \beta-1)}(x) \\
& -\frac{4(\beta-\alpha)(n+1)}{(2 n+\alpha+\beta)(2 n+\alpha+\beta+2)} P_{n+1}^{(\alpha-1, \beta-1)}(x) \\
& -\frac{4(n+1)(n+2)}{(2 n+\alpha+\beta+1)(2 n+\alpha+\beta+2)} P_{n+2}^{(\alpha-1, \beta-1)}(x) \text {, } \\
& P_{n}^{(\alpha, \beta)}(x)=\frac{(n+\alpha+\beta+2)(n+\alpha+\beta+1)}{(2 n+\alpha+\beta+2)(2 n+\alpha+\beta+1)} P_{n}^{(\alpha+1, \beta+1)}(x) \\
& -\frac{(\beta-\alpha)(n+\alpha+\beta+1)}{(2 n+\alpha+\beta)(2 n+\alpha+\beta+2)} P_{n-1}^{(\alpha+1, \beta+1)}(x) \\
& -\frac{(n+\alpha)(n+\beta)}{(2 n+\alpha+\beta)(2 n+\alpha+\beta+1)} P_{n-2}^{(\alpha+1, \beta+1)}(x) \text {. }
\end{aligned}
$$

The identity (B.4a $)$ is explained just above Eq. (I.4) in the main text. The identities (B.4b) and $(\overline{\mathrm{B} .4 \mathrm{~d}})$ can be derived by matching powers of $\frac{1-x}{2}$ in the hypergeometric representation of the Jacobi polynomial, Eq. (VI.2). The third identity, Eq. (B.4c), is [29, Eq. (22.7.1)]. The last identity, Eq. (B.4e $)$ can be derived using

$$
\begin{aligned}
P_{n}^{(\alpha, \beta)}(x) & =\frac{\Gamma(n+\alpha+1)}{n ! \Gamma(\alpha+1)}{ }_{2} F_{1}\left(-n, \alpha+\beta+n+1 ; \alpha+1 ; \frac{1-x}{2}\right) \\
& =\frac{\Gamma(n+\alpha+1)}{\Gamma(n+\alpha+\beta+1)} \sum_{p=0}^{n}(-1)^{p} \frac{\Gamma(\alpha+\beta+n+1+p)}{p !(n-p) ! \Gamma(\alpha+1+p)}\left(\frac{1-x}{2}\right)^{p} \\
& =\frac{\Gamma(n+\alpha+1)}{2^{n} \Gamma(n+\alpha+\beta+1)}(x-1)^{n} \sum_{p=0}^{n} \frac{2^{p} \Gamma(\alpha+\beta+2 n+1-p)}{p !(n-p) ! \Gamma(\alpha+1+n-p)(x-1)^{p}},
\end{aligned}
$$

and matching powers of $(x-1)$. The Jacobi polynomial identities (B.4) translate into the 
harmonic identities

$$
\begin{aligned}
& \partial_{\vartheta} Y_{j, j_{3}}(\vartheta, \phi)=\frac{\frac{\kappa}{2}-j_{3} \cos \vartheta}{\sin \vartheta} Y_{j, j_{3}}(\vartheta, \phi)-\sqrt{\left(j+j_{3}\right)\left(j-j_{3}+1\right)} e^{i \phi} Y_{j, j_{3}-1}(\vartheta, \phi), \\
& \partial_{\vartheta} Y_{j, j_{3}}(\vartheta, \phi)=\frac{j_{3} \cos \vartheta-\frac{\kappa}{2}}{\sin \vartheta} Y_{j, j_{3}}(\vartheta, \phi)+\sqrt{\left(j+j_{3}+1\right)\left(j-j_{3}\right)} e^{-i \phi} Y_{j, j_{3}+1}(\vartheta, \phi), \\
& \cos \vartheta Y_{j, j_{3}}(\vartheta, \phi)=-\frac{1}{2(j+1)} \sqrt{\frac{\left((j+1)^{2}-\frac{\kappa^{2}}{4}\right)\left((j+1)^{2}-j_{3}^{2}\right)}{\left(j+\frac{1}{2}\right)\left(j+\frac{3}{2}\right)}} Y_{j+1, j_{3}}(\vartheta, \phi) \\
& +\frac{\kappa j_{3}}{2 j(j+1)} Y_{j, j_{3}}(\vartheta, \phi) \\
& -\frac{1}{2 j} \sqrt{\frac{\left(j+\frac{\kappa}{2}\right)\left(j-\frac{\kappa}{2}\right)\left(j+j_{3}\right)\left(j-j_{3}\right)}{\left(j-\frac{1}{2}\right)\left(j+\frac{1}{2}\right)}} Y_{j-1, j_{3}}(\vartheta, \phi) \\
& \sin \vartheta e^{-i \phi} Y_{j, j_{3}}(\vartheta, \phi)=-\frac{1}{2(j+1)} \sqrt{\frac{\left((j+1)^{2}-\frac{\kappa^{2}}{4}\right)\left(j-j_{3}+2\right)\left(j-j_{3}+1\right)}{\left(j+\frac{1}{2}\right)\left(j+\frac{3}{2}\right)}} Y_{j+1, j_{3}-1}(\vartheta, \phi) \\
& +\frac{\kappa}{2 j(j+1)} \sqrt{\left(j+j_{3}\right)\left(j-j_{3}+1\right)} Y_{j, j_{3}-1}(\vartheta, \phi) \\
& +\frac{1}{2 j} \sqrt{\frac{\left(j+\frac{\kappa}{2}\right)\left(j-\frac{\kappa}{2}\right)\left(j+j_{3}\right)\left(j+j_{3}-1\right)}{\left(j-\frac{1}{2}\right)\left(j+\frac{1}{2}\right)}} Y_{j-1, j_{3}-1}(\vartheta, \phi), \\
& \sin \vartheta e^{i \phi} Y_{j, j_{3}}(\vartheta, \phi)=\frac{1}{2(j+1)} \sqrt{\frac{\left((j+1)^{2}-\frac{\kappa^{2}}{4}\right)\left(j+j_{3}+2\right)\left(j+j_{3}+1\right)}{\left(j+\frac{1}{2}\right)\left(j+\frac{3}{2}\right)}} Y_{j+1, j_{3}+1}(\vartheta, \phi) \\
& +\frac{\kappa}{2 j(j+1)} \sqrt{\left(j+j_{3}+1\right)\left(j-j_{3}\right)} Y_{j, j_{3}+1}(\vartheta, \phi) \\
& -\frac{1}{2 j} \sqrt{\frac{\left(j+\frac{\kappa}{2}\right)\left(j-\frac{\kappa}{2}\right)\left(j-j_{3}\right)\left(j-j_{3}-1\right)}{\left(j-\frac{1}{2}\right)\left(j+\frac{1}{2}\right)}} Y_{j-1, j_{3}+1}(\vartheta, \phi) .
\end{aligned}
$$

Applying these identities to Eq. (B.2), one finds, after some tedious algebra, that

$$
\begin{aligned}
\left\langle r, \vartheta, \phi \mid E ; 1 ; j-\frac{1}{2}, j, j_{3}\right\rangle= & \frac{1}{\sqrt{2 m E}}\left\{2 \frac{R_{-}^{\prime}-\frac{R_{-}}{r}\left(j-\frac{1}{2}\right)}{2 j+1} \sqrt{\left(j+\frac{1}{2}\right)^{2}-\frac{\kappa^{2}}{4}}\left\langle\vartheta, \phi \mid j+\frac{1}{2}, j, j_{3}\right\rangle\right. \\
& \left.+\left(\kappa \frac{R_{-}^{\prime}-\frac{R_{-}}{r}\left(j-\frac{1}{2}\right)}{2 j+1}-\theta R_{-}\right)\left\langle\vartheta, \phi \mid j-\frac{1}{2}, j, j_{3}\right\rangle\right\}, \\
\left\langle r, \vartheta, \phi \mid E ; 1 ; j+\frac{1}{2}, j, j_{3}\right\rangle= & \frac{1}{\sqrt{2 m E}}\left\{2 \frac{R_{+}^{\prime}+\frac{R_{+}}{r}\left(j+\frac{3}{2}\right)}{2 j+1} \sqrt{\left(j+\frac{1}{2}\right)^{2}-\frac{\kappa^{2}}{4}}\left\langle\vartheta, \phi \mid j-\frac{1}{2}, j, j_{3}\right\rangle\right. \\
& \left.-\left(\kappa \frac{R_{+}^{\prime}+\frac{R_{+}}{r}\left(j+\frac{3}{2}\right)}{2 j+1}+\theta R_{+}\right)\left\langle\vartheta, \phi \mid j+\frac{1}{2}, j, j_{3}\right\rangle\right\},
\end{aligned}
$$

where recall that

$$
R_{ \pm}=R_{E, j \pm \frac{1}{2}}(r)
$$

the radial dependence of the appropriate bosonic wavefunction, and the fermionic harmonics are given in Eq. (VI.6). 


\section{APPENDIX C: UNITARY REPRESENTATIONS OF SO(3,1) AND ITS CON- TRACTION}

In this section we review the unitary representations of $\mathrm{SO}(3,1)$ and of the Galilei group. For more details, see e.g. [30].

Consider the six dimensional algebra of hermitian operators,

$$
\left[J^{i}, J^{j}\right]=i \epsilon^{i j k} J^{k}, \quad\left[J^{i}, K^{j}\right]=i \epsilon^{i j k} K^{k}, \quad\left[K^{i}, K^{j}\right]=-i \alpha \epsilon^{i j k} J^{k}, \quad i=1,2,3, \quad \alpha \geq 0 .
$$

For $\alpha>0, K$ can be rescaled to obtain the standard $\mathrm{SO}(3,1)$ generators; $\alpha=0$ corresponds to the contracted algebra. So without loss of generality, one could restrict to $\alpha=0,1$.

The Casimirs of the algebra (C.1) are

$$
C_{1}=\alpha \vec{J}^{2}-\vec{K}^{2}, \quad C_{2}=\vec{J} \cdot \vec{K}
$$

Because of the subalgebra generated by $\vec{J}$, a unitary irreducible representation (irrep) is given by a sum of unitary $\mathrm{SU}(2)$ representations. So states in the unitary irrep are labelled

$$
\left|c_{1}, c_{2} ; j, j_{3}\right\rangle
$$

where $c_{1}$ and $c_{2}$ are the values of the Casimirs ( $\overline{C .2}$ ), and, of course, $j(j+1)$ is the value of the $\mathrm{SU}(2)$ Casimir $\vec{J}^{2}$ on the state, $j_{3}=-j,-j+1, \ldots, j-1, j$ is the eigenvalue of $J^{3}$ on the state, and $j$ is half a nonnegative integer.

Because $\vec{K}$ is a spin one operator, it will shift the value of $j$ at most by one. The $\left[K^{i}, J^{j}\right]$ commutators - or Clebsch-Gordan coefficients-yield

$$
\begin{aligned}
K^{3}\left|c_{1}, c_{2} ; j, j_{3}\right\rangle=- & \sqrt{j^{2}-j_{3}^{2}} A_{j-1, j}^{c_{1}, c_{2}}\left|c_{1}, c_{2} ; j-1, j_{3}\right\rangle+j_{3} A_{j, j}^{c_{1}, c_{2}}\left|c_{1}, c_{2} ; j, j_{3}\right\rangle \\
& +\sqrt{(j+1)^{2}-j_{3}^{2}} A_{j+1, j}^{c_{1}, c_{2}}\left|c_{1}, c_{2} ; j+1, j_{3}\right\rangle, \\
K^{+}\left|c_{1}, c_{2} ; j, j_{3}\right\rangle=- & \sqrt{\left(j-j_{3}\right)\left(j-j_{3}-1\right)} A_{j-1, j}^{c_{1}, c_{2}}\left|c_{1}, c_{2} ; j-1, j_{3}+1\right\rangle \\
& +\sqrt{\left(j-j_{3}\right)\left(j+j_{3}+1\right)} A_{j, j}^{c_{1}, c_{2}}\left|c_{1}, c_{2} ; j, j_{3}+1\right\rangle \\
& -\sqrt{\left(j+j_{3}+1\right)\left(j+j_{3}+2\right)} A_{j+1, j}^{c_{1}, c_{2}}\left|c_{1}, c_{2} ; j+1, j_{3}+1\right\rangle, \\
K^{-}\left|c_{1}, c_{2} ; j, j_{3}\right\rangle=\sqrt{\left(j+j_{3}\right)\left(j+j_{3}-1\right)} A_{j-1, j}^{c_{1}, c_{2}}\left|c_{1}, c_{2} ; j-1, j_{3}-1\right\rangle & +\sqrt{\left(j+j_{3}\right)\left(j-j_{3}+1\right)} A_{j, j}^{c_{1}, c_{2}}\left|c_{1}, c_{2} ; j, j_{3}-1\right\rangle \\
& +\sqrt{\left(j-j_{3}+1\right)\left(j-j_{3}+2\right)} A_{j+1, j}^{c_{1}, c_{2}}\left|c_{1}, c_{2} ; j+1, j_{3}-1\right\rangle,
\end{aligned}
$$

where the coefficients $A_{j^{\prime}, j}^{c_{1}, c_{2}}$ remain to be determined. By appropriately choosing the relative phases of $\left|c_{1}, c_{2} ; j, j_{3}\right\rangle$ and using $\left(K^{-}\right)^{\dagger}=K^{+}$, one can take

$$
A_{j, j}^{c_{1}, c_{2}} \in \mathbb{R}, \quad A_{j-1, j}^{c_{1}, c_{2}}=-A_{j, j-1}^{c_{1}, c_{2}} \geq 0 .
$$

Because $j \geq 0$, there is a minimum value, $j_{0}$, of $j$. Except for the trivial representation, $j=j_{0}=0$, there is no maximum value of $j$ because unitary irreps of noncompact algebras 
are infinite dimensional. All other values of $j$ in the irrep are obtained by applying $\vec{K}$ one or more times, and so typically $j=j_{0}, j_{0}+1, \ldots$ (The exceptions are the trivial singlet representation and the representations with $\vec{K}=0$ if $\alpha=0$.)

Evaluating the Casimir $C_{2}$ on a state, using Eq. (C.4) yields

$$
A_{j, j}^{c_{1}, c_{2}}=\frac{c_{2}}{j(j+1)} \text {. }
$$

(In matrix elements, this will be multiplied by zero if $j=0$ and so is effectively well-defined.) Similarly, the Casimir $C_{1}$ gives the less trivial relation

$$
\alpha j(j+1)-c_{1}=-j(2 j-1) A_{j-1, j}^{c_{1}, c_{2}} A_{j, j-1}^{c_{1}, c_{2}}+j(j+1)\left(A_{j, j}^{c_{1}, c_{2}}\right)^{2}-(j+1)(2 j+3) A_{j, j+1}^{c_{1}, c_{2}} A_{j+1, j}^{c_{1}, c_{2}} .
$$

The commutator $\left[K^{+}, K^{-}\right]=-2 \alpha J^{3}$ translates to

$$
-(2 j-1) A_{j-1, j}^{c_{1}, c_{2}} A_{j, j-1}^{c_{1}, c_{2}}+\left(A_{j, j}^{c_{1}, c_{2}}\right)^{2}+(2 j+3) A_{j+1, j}^{c_{1}, c_{2}} A_{j, j+1}^{c_{1}, c_{2}}=-\alpha \text { or } j=0 .
$$

Eqs. (C.7), (C.8), (C.6) and (C.5) combine to yield

$$
\left[A_{j, j+1}^{c_{1}, c_{2}}\right]^{2}=\frac{\alpha j(j+1)^{2}(j+2)-(j+1)^{2} c_{1}-c_{2}^{2}}{(j+1)^{2}(2 j+1)(2 j+3)} .
$$

In order that $j=j_{0}$ be the smallest value of $j$ that appears in the unitary irrep, it is necessary that $A_{j_{0}-1, j_{0}}^{c_{1}, c_{2}}=0$. Conversely, $A_{j-1, j}=0$ when

$$
\alpha j^{2}=\frac{1}{2}\left(c_{1}+\alpha\right) \pm \sqrt{\frac{1}{4}\left(c_{1}+\alpha\right)^{2}+c_{2}^{2}}
$$

From this it is deduced that

- For $c_{2} \neq 0$, only the plus sign gives a valid $j^{2}$, and so, as anticipated above, all $\mathrm{SU}(2)$ representations $j=j_{0}, j_{0}+1, \ldots$ appear in the unitary irrep. Moreover, $\alpha j_{0}^{2}=\frac{1}{2}\left(c_{1}+\alpha\right)+\sqrt{\frac{1}{4}\left(c_{1}+\alpha\right)^{2}+c_{2}^{2}}>0$; i.e. $j_{0}=0$ is incompatible with $c_{2} \neq 0$. Also, $c_{1}=\alpha\left(j_{0}^{2}-1\right)-\frac{c_{2}^{2}}{j_{0}^{2}}$.

- If $c_{2}=0$ then $A_{j-1, j}^{c_{1}, c_{2}}=0$ for $j=0$ or $\alpha j^{2}=\alpha+c_{1}$. Eq. (C.9) implies that $c_{1} \leq 0$ if $j_{0}=c_{2}=0$. Thus,

$-c_{1}=0=c_{2}$ is the singlet representation $j=j_{0}=0$, unless $\alpha=0$. If $\alpha=0$ then $c_{1}=0=c_{2}$ implies $j=j_{0}$, but $j_{0}$ may take any value. This corresponds to the partially trivial unitary irrep that is a unitary irrep of $\mathrm{SU}(2)$ with $\vec{K}=0$.

- If $j_{0}=0$ and $c_{1}<0\left(c_{2}=0\right)$, there is no positive integer value of $j$, except $j=j_{0}$, for which $A_{j-1, j}^{c_{1}, c_{2}}=0$. Thus, again $j=j_{0}, j_{0}+1, \ldots$

- If $j_{0}>0$ and $c_{2}=0$, then $c_{1}=\alpha\left(j_{0}^{2}-1\right)$. So if $\alpha=0$, then $c_{2}=0$ implies $c_{1}=0$, corresponding to the partially trivial irrep. 
Finally, it is convenient to write

$$
c_{2}=j_{0} \zeta, \quad \zeta \in \mathbb{R} .
$$

When $j_{0} \neq 0$, the above considerations imply

$$
c_{1}=\alpha\left(j_{0}^{2}-1\right)-\zeta^{2} .
$$

If $j_{0}=0$ (and therefore $c_{2}=0$ ), and $\alpha \neq 0$, then $c_{1}$ is arbitrary.

To summarize, if the Casimir

$$
C_{2}=\vec{J} \cdot \vec{K}=j_{0} \zeta \neq 0,
$$

then the representation consists of states with $j=j_{0}, j_{0}+1, \ldots$, and the other Casimir has value

$$
C_{1}=\alpha \vec{J}^{2}-\vec{K}^{2}=\alpha\left(j_{0}^{2}-1\right)-\zeta^{2} .
$$

This is the case needed in the main text; the other cases have $\vec{J} \cdot \vec{K}=0$.

\section{APPENDIX D: NORMALIZATION OF THE UNBOUND BOSONIC STATES}

In this section the computation of the normalization of the wavefunction (VI.21) is given. This is accomplished by computing the norm of the radial function

$$
R_{E, j}(r)=\frac{1}{r} M_{-i \frac{\kappa \theta}{\varepsilon}, j+\frac{1}{2}}(-i \varepsilon r), \quad \varepsilon \equiv \sqrt{2 m E-\theta^{2}}
$$

The first step in the calculation is the integral [28, Eq. (7.622.3)],

$$
\begin{gathered}
\int_{0}^{\infty} d x x^{\nu-1} e^{-b x} M_{\lambda_{1}, \mu_{1}-\frac{1}{2}}\left(a_{1} x\right) M_{\lambda_{2}, \mu_{2}-\frac{1}{2}}\left(a_{2} x\right)=a_{1}^{\mu_{1}} a_{2}^{\mu_{2}}\left[b+\frac{1}{2}\left(a_{1}+a_{2}\right)\right]^{-\nu-\mu_{1}-\mu_{2}} \Gamma\left(\nu+\mu_{1}+\mu_{2}\right) \\
\times F_{2}\left(\nu+\mu_{1}+\mu_{2} ; \mu_{1}-\lambda_{1}, \mu_{2}-\lambda_{2} ; 2 \mu_{1}, 2 \mu_{2} ; \frac{a_{1}}{b+\frac{1}{2}\left(a_{1}+a_{2}\right)}, \frac{a_{2}}{b+\frac{1}{2}\left(a_{1}+a_{2}\right)}\right), \\
\operatorname{Re}\left(\nu+\mu_{1}+\mu_{2}\right)>0, \operatorname{Re}\left(b \pm \frac{1}{2} a_{1} \pm \frac{1}{2} a_{2}\right)>0, \quad \text { (D.2a) }
\end{gathered}
$$

using Bailey's [31] notation for Appell's second hypergeometric function of two variables,

$$
F_{2}\left(\alpha ; \beta, \beta^{\prime} ; \gamma, \gamma^{\prime} ; x, y\right) \equiv \frac{\Gamma(\gamma) \Gamma\left(\gamma^{\prime}\right)}{\Gamma(\alpha) \Gamma(\beta) \Gamma\left(\beta^{\prime}\right)} \sum_{m, n=0}^{\infty} \frac{\Gamma(\alpha+n+m) \Gamma(\beta+n) \Gamma\left(\beta^{\prime}+m\right)}{n ! m ! \Gamma(\gamma+n) \Gamma\left(\gamma^{\prime}+m\right)} x^{n} y^{m}
$$

The reader who wishes to derive the integral (D.2a) can easily do so by writing the left-hand side in terms of the confluent hypergeometric series, and integrating term by term in $r$ to immediately obtain the double series $(\overline{\mathrm{D} .2 \mathrm{~b}})$. It will be possible to simplify the result by using the identity

$$
\begin{aligned}
& F_{2}\left(\alpha+1 ; \beta, \beta^{\prime} ; \alpha, \alpha ; x, y\right)=\frac{1}{\alpha\left(\beta-\beta^{\prime}\right)}(1-x-y)^{\alpha-\beta-\beta^{\prime}-1}(1-x)^{\beta^{\prime}-\alpha+1}(1-y)^{\beta-\alpha+1} \\
& \quad \times\left\{\beta \frac{\alpha-2 \beta^{\prime}-x\left(\alpha-\beta-\beta^{\prime}\right)}{1-x}{ }_{2} F_{1}\left(\alpha-\beta-1, \alpha-\beta^{\prime} ; \alpha ; \frac{x y}{(1-x)(1-y)}\right)\right. \\
& \left.\quad+\beta^{\prime} \frac{-\alpha+2 \beta+y\left(\alpha-\beta-\beta^{\prime}\right)}{1-y}{ }_{2} F_{1}\left(\alpha-\beta, \alpha-\beta^{\prime}-1 ; \alpha ; \frac{x y}{(1-x)(1-y)}\right)\right\} .
\end{aligned}
$$


The identity (D.3) is derived from the identity [31],

$$
F_{2}\left(\alpha ; \beta, \beta^{\prime} ; \alpha, \alpha ; x, y\right)=(1-x)^{-\beta}(1-y)^{-\beta^{\prime}} F_{1}\left(\beta, \beta^{\prime} ; \alpha ; \frac{x y}{(1-x)(1-y)}\right),
$$

which can be confirmed by writing the right-hand side as a power series in $x$ and $y$. In order to put the Appell function on the left-hand side of (D.3) into ones of the form of that on the left-hand side of (D.4), write

$$
\begin{aligned}
& \Gamma(\alpha+n+m+1) \Gamma(\beta+n) \Gamma\left(\beta^{\prime}+m\right) \\
& =\left[\left(\alpha-\beta-\beta^{\prime}\right)+(\beta+n)+\left(\beta^{\prime}+m\right)\right] \Gamma(\alpha+n+m) \Gamma(\beta+n) \Gamma\left(\beta^{\prime}+m\right) \\
& =\left(\alpha-\beta-\beta^{\prime}\right) \Gamma(\alpha+n+m) \Gamma(\beta+n) \Gamma\left(\beta^{\prime}+m\right)+\Gamma(\alpha+n+m) \Gamma(\beta+1+n) \Gamma\left(\beta^{\prime}+m\right) \\
& +\Gamma(\alpha+n+m) \Gamma(\beta+n) \Gamma\left(\beta^{\prime}+1+m\right), \quad(\mathrm{D} .5)
\end{aligned}
$$

which implies

$$
\begin{array}{r}
F_{2}\left(\alpha+1 ; \beta, \beta^{\prime} ; \alpha, \alpha ; x, y\right)=\frac{\alpha-\beta-\beta^{\prime}}{\alpha} F_{2}\left(\alpha ; \beta, \beta^{\prime} ; \alpha, \alpha ; x, y\right)+\frac{\beta}{\alpha} F_{2}\left(\alpha ; \beta+1, \beta^{\prime} ; \alpha, \alpha ; x, y\right) \\
+\frac{\beta^{\prime}}{\alpha} F_{2}\left(\alpha ; \beta, \beta^{\prime}+1 ; \alpha, \alpha ; x, y\right) . \quad(\mathrm{D} .6)
\end{array}
$$

The Appell functions on the right-hand side of (D.6) can then be written in terms of ordinary hypergeometric functions via Eq. (D.4). In fact, the identity,

$$
{ }_{2} F_{1}\left(\beta, \beta^{\prime} ; \alpha ; z\right)=\frac{\beta}{\beta-\beta^{\prime}}{ }_{2} F_{1}\left(\beta+1, \beta^{\prime} ; \alpha ; z\right)-\frac{\beta^{\prime}}{\beta-\beta^{\prime}}{ }_{2} F_{1}\left(\beta, \beta^{\prime}+1 ; \alpha ; z\right),
$$

whose derivation is similar to that of (D.6), allows one to write the hypergeometric function from the first term in Eq. (D.6) in terms of the other two. If one also uses [31]

$$
{ }_{2} F_{1}\left(\beta, \beta^{\prime} ; \alpha ; z\right)=(1-z)^{\alpha-\beta-\beta_{2}^{\prime}} F_{1}\left(\alpha-\beta, \alpha-\beta^{\prime} ; \alpha ; z\right), \quad \operatorname{Re} \gamma>\operatorname{Re} \alpha, \operatorname{Re} \beta>0,
$$

then one finally obtains (D.3).

With these formulas, and upon introducing a factor to ensure convergence of the integral, the inner product of interest is

$$
\begin{aligned}
N_{\varepsilon, \varepsilon^{\prime}, j}= & \lim _{\delta \rightarrow 0^{+}} \int_{0}^{\infty} d r r^{2} e^{-\delta r} R_{\varepsilon, j}(r) R_{\varepsilon^{\prime}, j}^{*}(r)=\lim _{\delta \rightarrow 0^{+}} \int_{0}^{\infty} d r e^{-\delta r} M_{-i \frac{\kappa \theta}{\varepsilon}, j+\frac{1}{2}}(-i \varepsilon r) M_{i \frac{\kappa \theta}{\varepsilon^{\prime}}, j+\frac{1}{2}}\left(i \varepsilon^{\prime} r\right) \\
= & \lim _{\delta \rightarrow 0^{+}} \frac{\delta}{\delta^{2}+\frac{1}{4}\left(\varepsilon-\varepsilon^{\prime}\right)^{2}} \frac{\left[\delta+\frac{i}{2}\left(\varepsilon-\varepsilon^{\prime}\right)\right]^{i \frac{\kappa \theta}{\varepsilon \varepsilon^{\prime}}\left(\varepsilon-\varepsilon^{\prime}\right)}}{\delta-\frac{i}{2}\left(\varepsilon-\varepsilon^{\prime}\right)} \frac{2(2 j+1) !\left(\varepsilon \varepsilon^{\prime}\right) j+1}{\left[\delta+\frac{1}{4}\left(\varepsilon+\varepsilon^{\prime}\right)^{2}\right]^{j}} \frac{\left.\left[\delta+\varepsilon^{\prime}\right)\right]^{i \frac{\kappa \theta}{\varepsilon}}}{\left[\delta+\frac{i}{2}\left(\varepsilon+\varepsilon^{\prime}\right)\right]^{i \frac{\kappa \theta}{\varepsilon^{\prime}}}} \\
& \times\left\{\frac{\varepsilon}{\varepsilon+\varepsilon^{\prime}} \frac{j+1+i \frac{\kappa \theta}{\varepsilon}}{\delta+\frac{i}{2}\left(\varepsilon+\varepsilon^{\prime}\right)}{ }_{2} F_{1}\left(j-i \frac{\kappa \theta}{\varepsilon}, j+1+i \frac{\kappa \theta}{\varepsilon^{\prime}} ; 2 j+2 ; \frac{\varepsilon \varepsilon^{\prime}}{\delta^{2}+\frac{1}{4}\left(\varepsilon+\varepsilon^{\prime}\right)^{2}}\right)\right. \\
& \left.+\frac{\varepsilon^{\prime}}{\varepsilon+\varepsilon^{\prime}} \frac{j+1-i \frac{\kappa \theta}{\varepsilon^{\prime}}}{\delta-\frac{i}{2}\left(\varepsilon+\varepsilon^{\prime}\right)}{ }_{2} F_{1}\left(j+1-i \frac{\kappa \theta}{\varepsilon}, j+i \frac{\kappa \theta}{\varepsilon^{\prime}} ; 2 j+2 ; \frac{\varepsilon \varepsilon^{\prime}}{\delta^{2}+\frac{1}{4}\left(\varepsilon+\varepsilon^{\prime}\right)^{2}}\right)\right\} .
\end{aligned}
$$


To proceed, observe that the first factor appears to imply the expected $\delta$-function, whereupon the hypergeometric functions are evaluated at unit argument. However, the denominator of the second factor provides a complication, which, fortunately, is overcome because it turns out that the leading-order contribution from the quantity in curly brackets vanishes. Thus, let us expand the quantity in curly brackets by using ${ }^{10}$

${ }_{2} F_{1}\left(\beta, \beta^{\prime} ; \alpha ; 1-z\right)=\frac{\Gamma(\alpha) \Gamma\left(\alpha-\beta-\beta^{\prime}\right)}{\Gamma(\alpha-\beta) \Gamma\left(\alpha-\beta^{\prime}\right)}\left[1-\frac{\beta \beta^{\prime}}{\alpha-\beta-\beta^{\prime}-1} z+\mathcal{O}\left(z^{2}\right)\right], \operatorname{Re}\left(\alpha-\beta-\beta^{\prime}\right)>1 ;$

we trust the reader will forgive us for applying this formula on the (excluded) boundary of the regime of validity. (The extra step (D.8) ensured that the parameters were at least on the boundary, and not completely outside the regime of validity of (D.10).)

Then,

$$
\begin{aligned}
N_{\varepsilon, \varepsilon^{\prime}, j}=\lim _{\delta \rightarrow 0^{+}} \frac{\left[\delta+\frac{i}{2}\left(\varepsilon-\varepsilon^{\prime}\right)\right]^{i \frac{\kappa \theta}{\varepsilon \varepsilon^{\prime}}\left(\varepsilon-\varepsilon^{\prime}\right)}}{\left[\delta^{2}+\frac{1}{4}\left(\varepsilon+\varepsilon^{\prime}\right)^{2}\right]^{j+1}} \frac{\left[\delta-\frac{i}{2}\left(\varepsilon+\varepsilon^{\prime}\right)\right]^{i \frac{\kappa \theta}{\varepsilon}}}{\left[\delta+\frac{i}{2}\left(\varepsilon+\varepsilon^{\prime}\right)\right]^{i \frac{}{\varepsilon^{\prime}}}} \frac{2(2 j+1) !^{2}\left(\varepsilon \varepsilon^{\prime}\right)^{j+1} \Gamma\left(1-i \frac{\kappa \theta}{\varepsilon \varepsilon^{\prime}}\left(\varepsilon-\varepsilon^{\prime}\right)\right)}{\Gamma\left(j+1+i \frac{\kappa \theta}{\varepsilon}\right) \Gamma\left(j+1-i \frac{\kappa \theta}{\varepsilon^{\prime}}\right)} \\
\quad \times\left\{\frac{\delta}{\delta^{2}+\frac{1}{4}\left(\varepsilon-\varepsilon^{\prime}\right)^{2}}-\frac{\delta}{\delta^{2}+\frac{1}{4}\left(\varepsilon+\varepsilon^{\prime}\right)^{2}} \frac{\left(j-i \frac{\kappa \theta}{\varepsilon}\right)\left(j+i \frac{\kappa \theta}{\varepsilon^{\prime}}\right)+j}{-i \frac{\kappa \theta}{\varepsilon \varepsilon^{\prime}}\left(\varepsilon-\varepsilon^{\prime}\right)}\right. \\
\left.+\frac{\delta^{2}+\frac{i}{2} \delta\left(\varepsilon-\varepsilon^{\prime}\right)}{\delta^{2}+\frac{1}{4}\left(\varepsilon-\varepsilon^{\prime}\right)^{2}} \frac{\delta}{\delta^{2}+\frac{1}{4}\left(\varepsilon+\varepsilon^{\prime}\right)^{2}} \frac{\varepsilon \varepsilon^{\prime}}{-i\left(\varepsilon-\varepsilon^{\prime}\right)}+\mathcal{O}\left(\delta\left[\delta+\frac{i}{2}\left(\varepsilon-\varepsilon^{\prime}\right)\right]\right)\right\} . \quad \text { (D.11) }
\end{aligned}
$$

By definition, $\varepsilon, \varepsilon^{\prime}>0$, so the denominator of the first factor is regular as $\delta \rightarrow 0^{+}$. It is the objects in the curly brackets that are interesting in the limit. The first term is a $\delta$-function. The second term vanishes in the limit. Splitting up the third term according to the numerator in the first factor, the $\delta^{2}$ term vanishes in the limit, and in the second term, the factor of $\left(\varepsilon-\varepsilon^{\prime}\right)$ cancels between numerator and the (last) denominator, leaving a $\delta$-function (and a factor of $-\frac{1}{2}$.) The neglected terms clearly vanish in the limit.

Since

$$
\lim _{\delta \rightarrow 0^{+}} \frac{\left[\delta-\frac{i}{2}\left(\varepsilon+\varepsilon^{\prime}\right)\right]^{\frac{\kappa \theta}{\varepsilon}}}{\left[\delta+\frac{i}{2}\left(\varepsilon+\varepsilon^{\prime}\right)\right]^{i \frac{\kappa \theta}{\varepsilon^{\prime}}}}=\frac{e^{-\frac{\pi i}{2} \frac{i \kappa \theta}{\varepsilon}}}{e^{\frac{\pi i}{2} \frac{\kappa \kappa \theta}{\varepsilon^{\prime}}}}=e^{\pi \frac{\kappa \theta}{2 \varepsilon \varepsilon^{\prime}}\left(\varepsilon+\varepsilon^{\prime}\right)}
$$

we have finally found

$$
N_{\varepsilon, \varepsilon^{\prime}, j}=\pi \frac{(2 j+1) !^{2}}{\left|\Gamma\left(j+1+i \frac{\kappa \theta}{\varepsilon}\right)\right|^{2}} e^{\pi \frac{\kappa \theta}{\varepsilon}} \delta\left(\varepsilon-\varepsilon^{\prime}\right), \quad \delta\left(\varepsilon-\varepsilon^{\prime}\right)=\frac{\sqrt{2 m E-\theta^{2}}}{m E} \delta\left(E-E^{\prime}\right) .
$$

[1] F. Denef, "Supergravity flows and D-brane stability," J. High Energy Phys. 08 (2000) 050; arXiv:hep-th/0005049v2.

10 This is a consequence of the identity $\frac{d_{2} F_{1}\left(\beta, \beta^{\prime} ; \alpha ; z\right)}{d z}=\frac{\beta \beta^{\prime}}{\alpha}{ }_{2} F_{1}\left(\beta+1, \beta^{\prime}+1 ; \alpha+1 ; z\right)$, which follows immediately from shifting the index of summation in the hypergeometric series, and from Gauss' formula [31] ${ }_{2} F_{1}\left(\beta, \beta^{\prime} ; \alpha ; 1\right)=\frac{\Gamma(\alpha) \Gamma\left(\alpha-\beta-\beta^{\prime}\right)}{\Gamma(\alpha-\beta) \Gamma\left(\alpha-\beta^{\prime}\right)}$. 
[2] F. Denef, "Quantum Quivers and Hall/Hole Halos," J. High Energy Phys. 10 (2002) 023; arXiv:hep-th/0206072.

[3] H. Sheinblatt, "Statistical entropy of an extremal black hole with 0-brane and 6-brane charge," Phys. Rev. D 57 (1998) 2421-2426; arXiv: hep-th/9705054.

[4] W. Taylor, "Adhering zero-branes to six-branes and eight-branes," Nucl. Phys. B508 (1997) 122-132; arXiv:hep-th/9705116.

[5] P. Claus et. al., "Black Holes and Superconformal Mechanics," Phys. Rev. Lett. 81 (1998) 4553-4556; arXiv:hep-th/9804177.

[6] J. Michelson and A. Strominger, "The Geometry of (Super) Conformal Quantum Mechanics," Commun. Math. Phys. 213 (2000) 1-17; arXiv:hep-th/9907191v3.

[7] J. Michelson and A. Strominger, "Superconformal Multi-Black Hole Quantum Mechanics," J. High Energy Phys. 09 (1999) 005; arXiv:hep-th/9908044v2.

[8] D. Gaiotto, A. Strominger and X. Yin, "Superconformal Black Hole Quantum Mechanics," J. High Energy Phys. 11 (2005) 017; arXiv:hep-th/0412322v1.

[9] S. Das et. al., "Branes wrapping Black Holes," Nucl. Phys. B733 (2006) 297-333; arXiv:hep-th/0507080.

[10] R. A. Coles and G. Papadopoulos, "The geometry of the one-dimensional supersymmetric non-linear sigma model," Class. Quant. Grav. 7 (1990) 427-438.

[11] D.-E. Diaconescu, R. Entin, "A Non-Renormalization Theorem for the $d=1, N=8$ Vector Multiplet," Phys. Rev. D 56 (1997) 8045-8052; arXiv:hep-th/9706059.

[12] E. D'Hoker and L. Vinet, "Supersymmetries of the Dyon," in Field Theory, Quantum Gravity and Strings, Vol. 2, H. J. De Vega and N. Sanchez, eds., (Springer-Verlag: 1987) p. 156-173.

[13] E. D'Hoker, V. A. Kostelecky and L. Vinet, "Spectrum Generating Superalgebras," in Dynamical Groups and Spectrum Generating Algebras, Vol. 1, A. Bohm et al., eds., (World Scientific: 1988) p. 339-367.

[14] E. D'Hoker and L. Vinet, "Hidden Symmetries Of A Spinning Particle In A Dyon Field," in Proceedings of the 14th International Colloquium on Group Theoretical Methods in Physics, Y. M. Cho, ed., (World Scientific: 1986) p. 576-582.

[15] E. D'Hoker and L. Vinet, "Spectrum (Super)Symmetries of Particles in a Coulomb Potential," Nucl. Phys. B260 (1985) 79.

[16] E. D'Hoker and L. Vinet, "Hidden Symmetries and Accidental Degeneracy for a Spin 1/2 Particle in the Field of a Dyon," Lett. Math. Phys. 12 (1986) 71.

[17] E. D'Hoker and L. Vinet, "Constants of Motion for a Spin 1/2 Particle in the Field of a Dyon," Phys. Rev. Lett. 55 (1985) 1043.

[18] P. A. Horvathy, "Dynamical (super)symmetries of monopoles and vortices," Rev. Math. Phys. 18 (2006) 329; arXiv:hep-th/0512233.

[19] L. Feher, P. A. Horvathy and L. O'Raifeartaigh, "Separating the Dyon System," Phys. Rev. D 40 (1989) 666.

[20] P. A. Horvathy and L. G. Feher, "Nonrelativistic Scattering of a Spin 1/2 Particle off a Selfdual Monopole," Mod. Phys. Lett. A3 (1988) 1451-1460.

[21] L. Feher, P. A. Horvathy and L. O'Raifeartaigh, "Applications Of Chiral Supersymmetry For Spin Fields In Selfdual Backgrounds," Int. J. Mod Phys. A4 (1989) 5277-5285.

[22] H. V. McIntosh and A. Cisneros, "Degeneracy in the Presence of a Magnetic Monopole," J. Math. Phys. 11 (1970) 896.

[23] F. Bloore and P. A. Horvathy, "Helicity-Supersymmetry of Dyons," J. Math. Phys. 33 (1992) 1869; arXiv:hep-th/0512144. 
[24] P. A. Horvathy, "The Biedenharn Approach to Relativistic Coulomb-type Problems," Rev. Math. Phys. 18 (2006) 311; arXiv:hep-th/0601123.

[25] E. Ivanov, S. Krisonos, and O. Lechtenfeld, "New Variant of $N=4$ Superconformal Mechanics," J. High Energy Phys. 03 (2003) 014; arXiv: hep-th/0212303.

[26] T. T. Wu and C. N. Yang, "Dirac Monopole Without Strings: Monopole Harmonics," Nucl. Phys. B107 (1976) 365-380.

[27] H. Poincaré, "Remarques sur une expérience de M. Birkeland," C. R. Acad. Sci., Paris 123 (1896) 530-533.

[28] I. S. Gradshteyn and I. M. Ryzhik, Table of Integrals, Series, and Products, Fifth Edition, (Academic Press: 1994).

[29] M. Abramowitz and I. A. Stegun, Handbook of Mathematical Functions with Formulas, Graphs, and Mathematical Tables, (Dover Publications: 1965).

[30] I. M. Gel'fand, R. A. Minlos and Z. Ya. Shapiro, Representations of the rotation and Lorentz groups and their applications, (The MacMillan Company: 1963).

[31] W. N. Bailey, Generalized Hypergeometric Series, (Stechart-Hafner Service Agency, Inc.: 1964). 